\title{
The nonlinear Schrödinger equation for the delta-comb potential: quasi-classical chaos and bifurcations of periodic stationary solutions
}

\author{
$D$ WITTHAUT ${ }^{a}, K$ RAPEDIUS $^{b}$ and $H \mathrm{~J} \mathrm{KORSCH}^{c}$ \\ ${ }^{a}$ QUANTOP, The Niels Bohr Institute, University of Copenhagen, 2100 Copenhagen, \\ Denmark \\ E-mail: dirk.witthaut@nbi.dk \\ ${ }^{b}$ FB Physik, Technical University of Kaiserslautern, D-67653 Kaiserslautern, Germany \\ E-mail: rapedius@physik.uni-kl.de \\ ${ }^{c}$ FB Physik, Technical University of Kaiserslautern, D-67653 Kaiserslautern, Germany \\ E-mail: korsch@physik.uni-kl.de
}

\begin{abstract}
The nonlinear Schrödinger equation is studied for a periodic sequence of deltapotentials (a delta-comb) or narrow Gaussian potentials. For the delta-comb the time-independent nonlinear Schrödinger equation can be solved analytically in terms of Jacobi elliptic functions and thus provides useful insight into the features of nonlinear stationary states of periodic potentials. Phenomena well-known from classical chaos are found, such as a bifurcation of periodic stationary states and a transition to spatial chaos. The relation of new features of nonlinear Bloch bands, such as looped and period doubled bands, are analyzed in detail. An analytic expression for the critical nonlinearity for the emergence of looped bands is derived. The results for the deltacomb are generalized to a more realistic potential consisting of a periodic sequence of narrow Gaussian peaks and the dynamical stability of periodic solutions in a Gaussian comb is discussed.
\end{abstract}

\section{Introduction}

In the case of low temperatures, the dynamics of a Bose-Einstein condensate (BEC) can be described in a mean-field approach by the nonlinear Schrödinger equation (NLSE) or Gross-Pitaevskii equation (see, e.g., [51,52])

$$
\left(-\frac{\hbar^{2}}{2 M} \nabla^{2}+V(x)+g|\psi(x, t)|^{2}\right) \psi(x, t)=\mathrm{i} \hbar \frac{\partial \psi(x, t)}{\partial t},
$$

where $g$ is the nonlinear interaction strength. Another important application of the nonlinear Schrödinger equation is the propagation of electromagnetic waves in nonlinear media (see, e.g., [23], chapter 8). 
D Witthaut, K Rapedius and H J Korsch

Due to the possibility to control all experimental parameters accurately over wide ranges and monitor the dynamics of a BEC in situ, these have become one of the most prominent models for the study of nonlinear dynamical systems. An increasing number of important nonlinear phenomena has been demonstrated experimentally in the last decade, such as the motion of dark [10] and bright solitons [34] and the self-trapping effect [4]. Furthermore, it is possible to reduce the dimensionality of the NLSE in confined geometries (see, e.g., [29] and references therein). An excellent review of nonlinear phenomena in BECs can be found in the recent article by Carretero-González et al. [13]. A particular important subdiscipline is the study of the nonlinear dynamics of ultracold atoms and BECs in periodic potentials since these systems provide excellent model systems for fundamental problems in condensed matter physics (see [45] and [6] for recent reviews). Among other phenomena, recent experiments have demonstrated the excistence of gap solitons [24] and of looped Bloch bands and the corresponding dynamical instability [25,33] as well as nonlinear de- and rephasing [47,68].

In this paper paper we will present an analytic study of stationary states of the nonlinear Schrödinger equation, i.e. states of the form $\psi(x, t)=\exp (-\mathrm{i} \mu t / \hbar) \psi(x)$, in a onedimensional periodic potential. These states satisfy the time-independent NLSE

$$
\left(-\frac{\hbar^{2}}{2 M} \frac{\mathrm{d}^{2}}{\mathrm{~d} x^{2}}+V(x)+g|\psi(x)|^{2}\right) \psi(x)=\mu \psi(x)
$$

with $V(x+d)=V(x)$ and $\mu \in \mathbb{R}$. Here we adopt a novel approach to nonlinear stationary states by exploiting the analogy to nonlinear dynamical systems in considering the wave function $\psi(x)$ as a dynamical variable. Although a bit unusual, this approach leads to a variety of important results, explaining several phenomena umambigiously in the language of nonlinear dynamical systems. For instance, the appearance of period-doubled Bloch bands, as described previously in [40], is readily understood as a familiar period-doubling bifurcation. Similarly, the bifurcation leading to the appearance of looped Bloch bands is easily understood so that it is possible to calculate the critical nonlinearity for this process analytically. From a fundamental point of view it becomes clear that Bloch states, i.e. solutions which are periodic up to a phase factor $e^{i \kappa d}$, are extremely fragile objects. While in the linear case $g=0$ all states are Bloch states or linear combinations of degenerate Bloch states according to Bloch's theorem, these states are of measure zero in the nonlinear case $g \neq 0$. The reason for this difference is that almost all solutions are only quasi-periodic for $g \neq 0$, approaching a true periodicity smoothly in the limit $g \rightarrow 0$. With increasing strength of the nonlinearity, more and more (quasi)-periodic orbits are lost and chaos sets in in the wake of a period doubling process. Spatial chaoticity usually implies a divergence of the wave function. Thus, well-behaved nonlinear stationary states are very rare for certain parameter values, in fact large interaction constants.

As a convenient model for a space-periodic structure, we consider a particular simple model system, the delta-comb potential

$$
V(x)=\lambda \sum_{n} \delta(x-d n)=\lambda \delta_{d}(x)
$$

The potential (1.3) has the great advantage that analytical solutions of the NLSE can be constructed in terms of Jacobi elliptic functions [11]. Analytic solutions of the nonlinear 
Schrödinger equation for a non-vanishing potential $V(x)$ are very rare and hence it will be of interest to study such a potential in some detail. Its linear counterpart represents a basic model for a quantum mechanical Bloch system (see, e.g. [30]). A generalization to a more realistic setup consisting of a periodic series of Gaussian potential barriers will be discussed at the end of this paper. Narrow Gaussian potential barriers can be realized in a good approximation in current atom-chip experiments (see [27] for a recent review). For example the resonant transport of BECs through two such peaks is analyzed in [49]. We generally restrict ourselves to the case of a repulsive interaction, $g>0$ and $\mu>0$. Some differences that arise for an attractive interaction will be discussed briefly in Sec. 2.4. For convenience we set $\hbar=M=1$ and fix the period of the potential as $d=2 \pi$. If the normalization of the wave function is arbitrary, one can rescale the wave function as $\psi \rightarrow \psi / \sqrt{g}$, leaving two free parameters $(\lambda, \mu)$, otherwise three parameters must be considered.

Up now, quite a number of articles were devoted to the important problem of nonlinear stationary states in a periodic potential and we refer to the review articles $[13,45]$ for an overview. There also have been some papers devoted to the NLSE with a delta-comb potential, however focusing on completly different aspects. Bound states were studied in [60] and the nonlinear transport problem through such a series of barriers was discussed in [59]. Bloch bands were studied in $[22,38,57,58]$, however relying mostly on numerical approaches. Recently, Paul et al. used a randomized delta-comb potential to study the crossover to Anderson localization in [50] in ultracold gases. A related dynamical approach to nonlinear eigenstates can be found in [53], however without taking much benefit from the theory of nonlinear Bloch bands. It should also be noted that bound states of nonlinear Schrödinger equations with a nonlinear periodic microstructure where $g=g(x)$ is spaceperiodic have been explored [26,64].

In particular this paper is organized as follows. The stationary solutions of the free NLSE (i.e. $V(x)=0$ ) are reviewed in Sec. 2 to lay the foundation for our further work. With these results in mind we then construct the solutions for the delta-comb potential in terms of Jacobi elliptic function in Sec. 2.2. thus reducing the NLSE to a discrete mapping for their parameters. In Sec. 3 we focus on periodic solutions and derive conditions for their existence. The stability of these solutions is discussed in detail in Sec. 3.2, We then apply our results to the important problem of nonlinear Bloch bands in Sec. 4, deriving the critical nonlinearity for their emergence analytically. Finally, we generalize our approach to a more realistic setup consisting of a periodic series of Gaussian potential barriers in Sec. 5 . In addition we discuss the temporal stability of nonlinear periodic solutions, showing that the stability properties are fundamentally different for different types of nonlinear Bloch states. A summary and discussion of our results is presented in Sec. 6

\section{Solutions of the nonlinear Schrödinger equation}

Before discussing particular solutions, we start with some general remarks on the timeindependent nonlinear Schrödinger equation (NLSE)

First, we note that the real-valued time-independent NLSE is mathematically equivalent to a classical nonlinear oscillator described by the nonlinear Hill equation

$$
\ddot{y}+f(t) y+\beta y^{3}=0, \quad f(t+T)=f(t) .
$$


Replacing the amplitude $y(t)$ by the wave function $\psi(x)$ and the time $t$ by the position variable $x$ and identifying $\beta=-2 g$ and $f(t)=2(\mu-V(x))$ one recovers the time-independent NLSE (1.2). For $V(x)=0$ one recovers the undamped Ueda oscillator (see, e.g., [36,44,48] and references therein). Within the framework of nonlinear eigenstates of BECs, this equation has been analyzed in [11]. For a cosine-potential $V(x)=V_{0} \cos (x)$, Eqn. (2.1) is just the nonlinear Matthieu equation, which is a popular example in classical nonlinear dynamics (see, e.g., $[7,19,43,48]$ ). The NLSE with a delta-comb potential $V(x)=\lambda \delta_{d}(x)$, or analogously the kicked nonlinear Hill equation, studied in the present paper has the advantage that it allows analytical solutions. Further exactly solvable examples can be found when the potential is given in terms of Jacobi elliptic functions $[9,31]$.

In quantum systems, however, we also encounter complex solutions of the NLSE discussed in sections 4 and 5 in the context of nonlinear Bloch bands and the temporal evolution of a wave function, which is governed by the time-dependent NLSE.

Furthermore, the time-independent NLSE (1.2) can be written as a Hamiltonian system with the conjugate variables $\left(\psi^{*}, \psi^{\prime}\right)$ and $\left(\psi, \psi^{\prime *}\right)$, where $\psi^{\prime}=\mathrm{d} \psi / \mathrm{d} x$. Introducing the Hamiltonian function

$$
\mathcal{H}=\left|\psi^{\prime}\right|^{2}-2(V(x)-\mu)|\psi|^{2}-g|\psi|^{4},
$$

Hamilton's equations read

$$
\begin{aligned}
\frac{\mathrm{d}}{\mathrm{d} x} \psi^{\prime} & =-\frac{\partial \mathcal{H}}{\partial \psi^{*}}=2(V(x)-\mu) \psi+2 g|\psi|^{2} \psi=\psi^{\prime \prime} \\
\frac{\mathrm{d}}{\mathrm{d} x} \psi^{*} & =\frac{\partial \mathcal{H}}{\partial \psi^{\prime}}=\psi^{\prime *}
\end{aligned}
$$

and analogously for $\left(\psi, \psi^{\prime *}\right)$. Considering only real-valued solutions, one is left with a two-dimensional phase space $\left(\psi, \psi^{\prime}\right)$ where the flow is is area-preserving.

For the special case of a delta-comb,

$$
V(x)=\lambda \delta_{2 \pi}(x)=\lambda \sum_{n} \delta(x-2 \pi n),
$$

the solutions are essentially the ones of the free NLSE. Hence we give a brief review of the real solutions of the free nonlinear Schrödinger equation in the following section. The first derivative of the wave function $\psi^{\prime}$ is discontinuous at $x=2 \pi n$ (cf. the studies $[2,20,54,56,67]$ of a NLSE for a single delta-potential)

$$
\lim _{\epsilon \rightarrow 0+}\left(\psi^{\prime}(2 \pi n+\epsilon)-\psi^{\prime}(2 \pi n-\epsilon)\right)=2 \lambda \psi(2 \pi n)
$$

whereas the wave function itself is continuous. The discontinuity of the delta-comb potential does not affect the area-preserving quality of the flow $\left(\psi\left(x_{0}\right), \psi^{\prime}\left(x_{0}\right)\right) \rightarrow\left(\psi(x), \psi^{\prime}(x)\right)$. To clarify this issue, we linearize the flow in the vicinity of a delta peak at $x=2 \pi n$ :

$$
\left(\begin{array}{c}
\psi(2 \pi n+\epsilon) \\
\psi^{\prime}(2 \pi n+\epsilon)
\end{array}\right)=\left(\begin{array}{cc}
1 & 0 \\
2 \lambda & 1
\end{array}\right)\left(\begin{array}{c}
\psi(2 \pi n-\epsilon) \\
\psi^{\prime}(2 \pi n-\epsilon)
\end{array}\right) .
$$

As the determinant of the matrix equals unity, the flow is clearly area-preserving. 

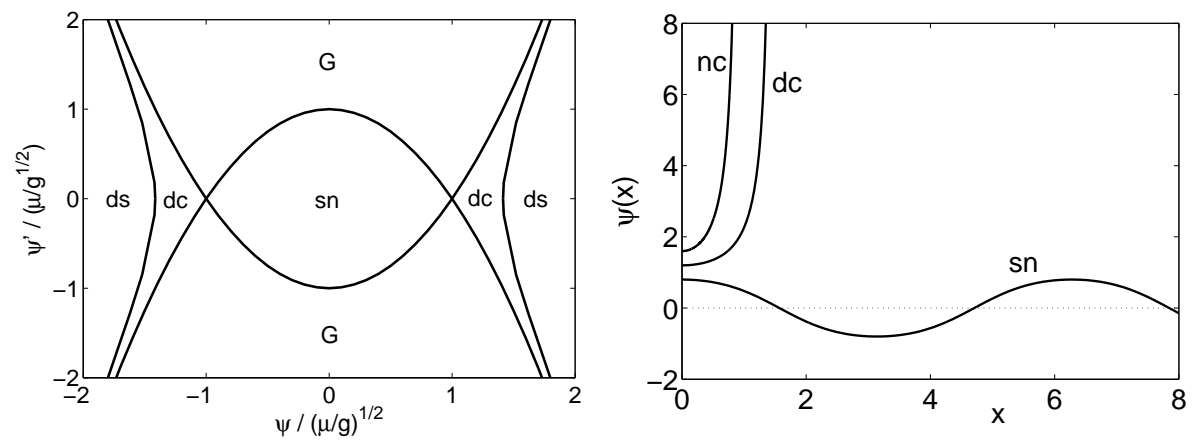

Figure 1. Solution types of the free nonlinear Schrödinger equation with a repulsive nonlinearity g. Left panel: Dependence of the solution type on the initial condition $\left(\psi\left(x_{0}\right), \psi^{\prime}\left(x_{0}\right)\right)$. Right panel: Examples of the solution types for $\psi^{\prime}\left(x_{0}\right)=0$ and $\psi\left(x_{0}\right)=0.8(\mathrm{sn}), 1.2$ (dc), 1.8 (nc) and $\mu=g=1$.

\subsection{Real solutions of the free nonlinear Schrödinger equation}

The free nonlinear Schrödinger equation has well known real solutions in terms of Jacobi elliptic functions $[11,21]$ (see, e.g., [1,37] for an introduction).

It will be instructive to calculate the general solution of the free NLSE explicitly. Multiplying Eqn. (1.2) for $V(x)=0$ by $\psi^{\prime}$ and integrating once yields the general solution of the free NLSE in the form

$$
x-x_{0}=\int_{\psi\left(x_{0}\right)}^{\psi(x)} \frac{\mathrm{d} s}{\sqrt{g s^{4}-2 \mu s^{2}+2 E}} .
$$

The type of solution of Eqn. (2.8) strongly depends on the initial values $\psi\left(x_{0}\right)$ and $\psi^{\prime}\left(x_{0}\right)$, which also determine the constant of integration $E$ via

$$
E=\frac{1}{2} \psi^{\prime}\left(x_{0}\right)^{2}-\frac{g}{2} \psi\left(x_{0}\right)^{4}+\mu \psi\left(x_{0}\right)^{2} .
$$

This dependence on the initial values is illustrated in Fig. 1. For initial values of $\psi\left(x_{0}\right)$ and $\psi^{\prime}\left(x_{0}\right)$ inside the regions marked by sn, dc and nc, the integral in Eqn. (2.8) can be reduced to the canonical form of the Jacobi elliptic functions sn, dc and nc, respectively. For, e.g., $\psi^{\prime}\left(x_{0}\right)=0$ a simple scaling of the wave function $\tilde{\psi}=\sqrt{g / \mu} \psi$ and the position $\tilde{x}=\sqrt{\mu} x$ leads directly to the standard forms given in [37], Chapter 3. Examples of these solutions types are shown on the right-hand side of Fig. 1. Note that only the sn-type solution is periodic while all other types diverge at a finite value of $x$.

The situation is more involved for initial values in the regions marked by G in Fig. 1 . A simple scaling is not sufficient any longer, but a transformation $t=(s+q) /(s-q)$ with $q=(2 E / g)^{1 / 4}$ brings the integral (2.8) to the standard form of the sc function [37].

Note that the sn-type solutions are periodic, while all other solutions (nc, dc and G) diverge at a finite value of $x$. This is easily understood in terms of the classical analog, the nonlinear Hill equation (2.1). This equation describes a classical particle with coordinate $y$ and kinetic energy $T=\dot{y}^{2} / 2$ moving in a potential $U(y)=\mu y^{2}+\beta y^{4} / 4$ with $\beta=-2 g$, that is illustrated in Fig. 2. For $g>0$ this potential has a minimum at $y=0$ and 


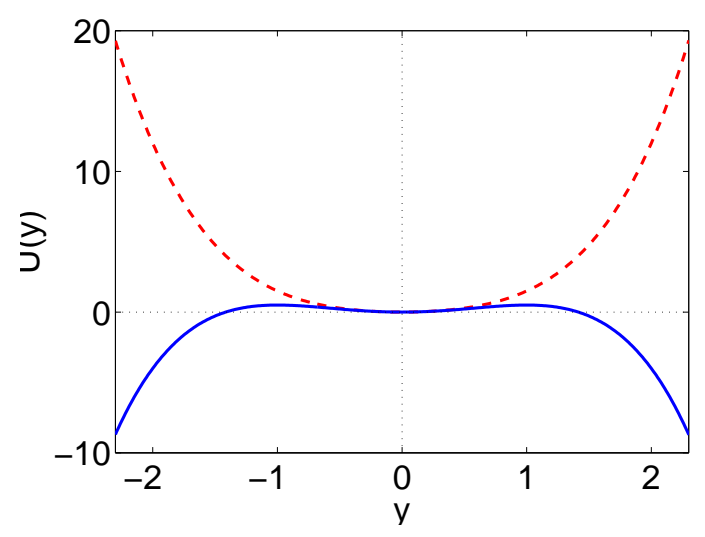

Figure 2. Classical potential $U(y)$ describing the nonlinear Hill equation resp. undamped Ueda equation (2.1) for $\mu=1$ and $g=1$ (-) resp. $g=-1(---)$.

diverges to $-\infty$ for $|y| \rightarrow \infty$. Thus $y$ will diverge as soon as the particle can leave the potential minimum around $y=0$. In contrast, the motion is always bounded for $g<0$. The different regions in Fig. 1 are directly related to the turning points for a system with energy $E=T+U=\dot{y}^{2} / 2+\mu y^{2}+\beta y^{4} / 4$. For $g>0$ and energies in the interval $0<E<U_{\max }=\mu^{2} / 2 g$, we have four real valued turning points with bounded trajectories in the sn-region and unbounded ones in the dc-region. For $E<0$, we have two real and two complex turning points and in the G-region for $E>U_{\max }$ all turning points are complex. The curves in Fig. 1 are the boundaries of these regions, i.e. $E=U_{\max }$ and $E=0$.

In the following we focus on the sn-type solutions since they do not diverge. For fixed values of $\mu$ and $g$ these solutions are explicitly given by

$$
\psi(x)=A \operatorname{sn}\left(4 K(p) \frac{x+x_{0}}{L} \mid p\right)
$$

where $p(0 \leq p \leq 1)$ denotes the elliptic parameter and $L$ is the period of the wave function. The amplitude $A$ and the period $L$ are fixed by

$$
A^{2}=\frac{2 \mu p}{g(p+1)} \quad \text { and } \quad L^{2}=\frac{8(p+1) K(p)^{2}}{\mu}
$$

where $K(p)$ denotes the complete elliptic integral of the first kind. However, it should be kept in mind that other types of solutions are appropriate for different initial values as illustrated in Fig. 1 .

\section{$2.2 \quad$ Non-diverging solutions for a delta-comb}

The real solutions of the nonlinear Schrödinger equation for a delta comb are essentially the ones of the free equation discussed above. Hence we make the ansatz

$$
\psi(x)=A_{n} \operatorname{sn}\left(4 K\left(p_{n}\right) \frac{x+x_{n}}{L_{n}} \mid p_{n}\right)
$$

for $x \in(2 \pi n, 2 \pi(n+1))$ with $A_{n}$ and $L_{n}$ defined in Eqn. (2.11). However, two conditions have to be fulfilled at $x=2 \pi n$ : The wave function is continuous, whereas its derivative 

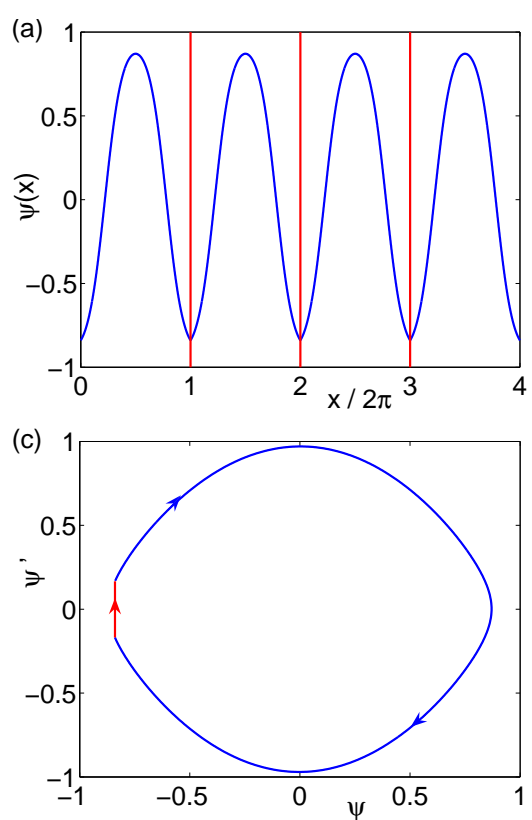
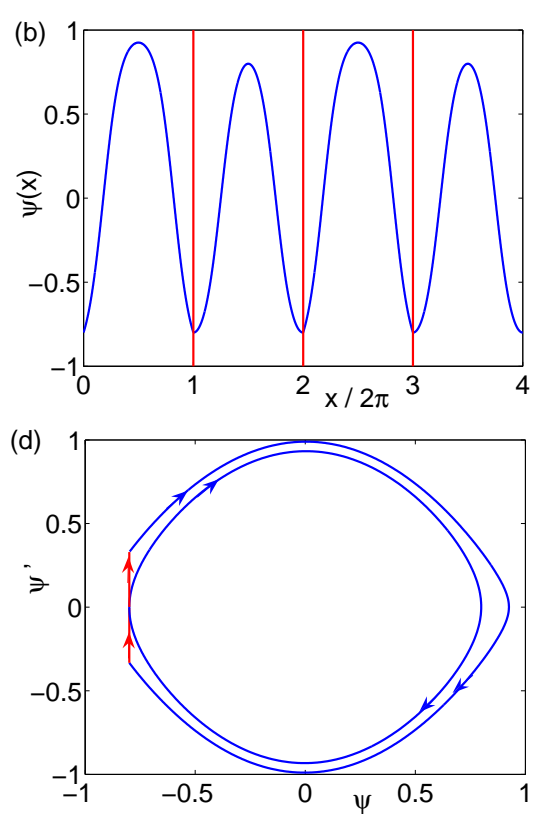

Figure 3. Periodic trajectories are given by solutions of the free nonlinear Schrödinger equation connected by vertical jumps caused by the delta potentials. The figure shows the 'configuration space' representation $\psi(x)(\mathrm{a}, \mathrm{b})$ as well as the phase space representation $\left(\psi, \psi^{\prime}\right)(\mathrm{c}, \mathrm{d})$. The period-one orbit for $\lambda=-0.2(\mathrm{a}, \mathrm{c})$ bifurcates to a period-two orbit (b,d) if the strength of the delta-potentials is changed to $\lambda=-0.205$. The remaining parameters are $g=\mu=1$.

is discontinuous according to Eqn. (2.6). This is illustrated in Fig $3(a, b)$, where the discontinuity of the the first derivative at $x=2 \pi n$ is clearly visible. For the sn-type solutions (2.12) the (dis-) continuity conditions are explicitly given by:

$$
\begin{aligned}
& \text { I. } A_{n} \operatorname{sn}\left(u_{n} \mid p_{n}\right)=A_{n+1} \operatorname{sn}\left(u_{n+1} \mid p_{n+1}\right) \\
& \text { II. } 2 \lambda A_{n} \operatorname{sn}\left(u_{n} \mid p_{n}\right)+\frac{4 A_{n} K_{n}}{L_{n}} \operatorname{cn}\left(u_{n} \mid p_{n}\right) \operatorname{dn}\left(u_{n} \mid p_{n}\right) \\
& \qquad=\frac{4 A_{n+1} K_{n+1}}{L_{n+1}} \operatorname{cn}\left(u_{n+1} \mid p_{n+1}\right) \operatorname{dn}\left(u_{n+1} \mid p_{n+1}\right)
\end{aligned}
$$

where the abbreviations $u_{n}=4 K\left(p_{n}\right)\left(2 \pi(n+1)+x_{n}\right) / L_{n}$ and $u_{n+1}=4 K\left(p_{n+1}\right)(2 \pi(n+$ 1) $\left.+x_{n+1}\right) / L_{n+1}$ have been used. If $\left|A_{n} \operatorname{sn}\left(u_{n} \mid p_{n}\right)\right| \leq\left|A_{n+1}\right|$ the first condition can always be fulfilled by an appropriate choice of the "phase shift" $x_{n+1}$. Inserting the first condition into the second one and using the addition theorems of the Jacobi elliptic functions one arrives at

$$
\begin{aligned}
& \frac{p_{n+1}}{\left(p_{n+1}+1\right)^{2}}=\frac{p_{n}}{\left(p_{n}+1\right)^{2}}+\frac{2 \lambda^{2}}{\mu} \frac{p_{n}}{p_{n}+1} \operatorname{sn}^{2}\left(u_{n} \mid p_{n}\right) \\
& +\frac{p_{n}}{\left(p_{n}+1\right)^{3 / 2}} \frac{4 \lambda}{\sqrt{2 \mu}} \operatorname{cn}\left(u_{n} \mid p_{n}\right) \operatorname{dn}\left(u_{n} \mid p_{n}\right) \operatorname{sn}\left(u_{n} \mid p_{n}\right) .
\end{aligned}
$$


These equations define a discrete mapping $f:\left(p_{n}, x_{n}\right) \rightarrow\left(p_{n+1}, x_{n+1}\right)$ or $\tilde{f}:\left(\psi_{n}, \psi_{n}^{\prime}\right) \rightarrow$ $\left(\psi_{n+1}, \psi_{n+1}^{\prime}\right)$, respectively, where we introduced the abbreviations $\psi_{n}=\psi(2 \pi n+0)$ and $\psi_{n}^{\prime}=\psi^{\prime}(2 \pi n+0)$ for convenience. In the following we will mainly consider the mapping $\tilde{f}$ in terms of the physical variables $\psi$ and $\psi^{\prime}$. This is the Poincare section of the areapreserving phase space flow generated by the NLSE. However, the mapping $f$ in terms of the abstract parameters $p_{n}$ and $x_{n}$ is more suitable for actual calculations.

Note that the discontinuity of the derivative may lead to a divergence of the wave function. As stated above, the sn-type solutions are periodic, while all other solutions of the free NLSE diverge at a finite value of $x$. Due to the discontinuity of the derivative, the mapping $\tilde{f}$ can map a point $\left(\psi_{n}, \psi_{n}^{\prime}\right)$ inside the sn-region of phase space to a point $\left(\psi_{n+1}, \psi_{n+1}^{\prime}\right)$ outside the sn-region. This will generally lead to a divergence of $\psi(x)$ at a finite value of $x$. We will come back to the question of divergences later in this section.
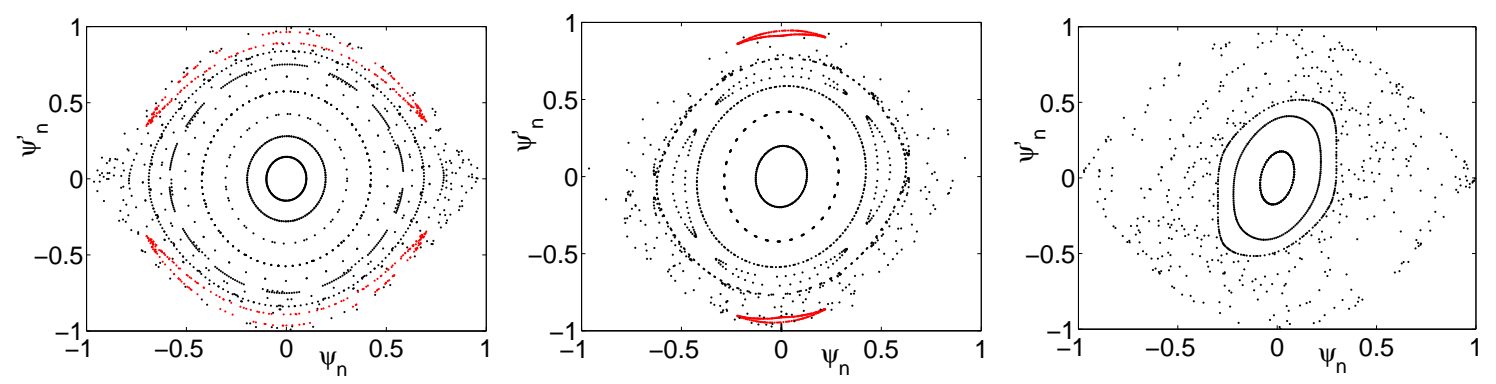

Figure 4. Stroboscopic phase space plots of the mapping $\tilde{f}:\left(\psi_{n}, \psi_{n}^{\prime}\right) \rightarrow\left(\psi_{n+1}, \psi_{n+1}^{\prime}\right)$ for $g=1$, $\mu=1$ and $\lambda=0.02,0.1,0.5$ (from left to right).

\subsection{Dynamics in phase space}

It is instructive to first have a look at the flow in phase space as illustrated in Fig. 3 (c,d) for periodic solutions $(g=1, \mu=1)$ in comparison to the respective wave function representation $(\mathrm{a}, \mathrm{b})$. Panel $(\mathrm{c})$ show a period-one trajectory following an orbit of the free nonlinear system as discussed in Sec. 2.1 up to a point $\psi \approx-0.84$ where the deltapotential causes a vertical jump of $\psi^{\prime}$ according to (2.6). If the potential strength $\lambda$ is made more negative, the orbit slightly adjusts until it bifurcates into a double-periodic orbit as shown in Fig. 3 (d) for $\lambda=-0.205$. Here two kicks connect two different orbits of the free nonlinear system. We will come back to the period-doubling bifurcation scenario in Sect. 3.2 (compare in particular Fig. 11). Similar phase space plots can be found in Ref. [5, 32,35].

We now turn to the Poincaré section of this this flow given by the discrete mapping $f$ or $\tilde{f}$ respectively, because it offers a global view of the dynamics. For the actual computation we use the mapping $f$ in terms of $p_{n}$ and $x_{n}$, in fact the Eqn. (2.15). We propagate an ensemble of trajectories with randomly chosen initial conditions within the sn-region of phase space (cf. Fig. 1). The dynamics within this region is visualized in Figs. 4 and 5 for different values of the potential strength $\lambda$, both for repulsive and attractive deltapotentials. The remaining parameters are fixed as $\mu=1$ and $g=1$. First we notice that the dynamics is invariant with respect to a global sign change $\left(\psi_{n}, \psi_{n}^{\prime}\right) \rightarrow\left(-\psi_{n},-\psi_{n}^{\prime}\right)$, hence the phase space is inversion symmetric. For $\lambda=0$ the phase space is additionally 
symmetric to the coordinate axes. This symmetry is destroyed with increasing potential strength $\lambda$.
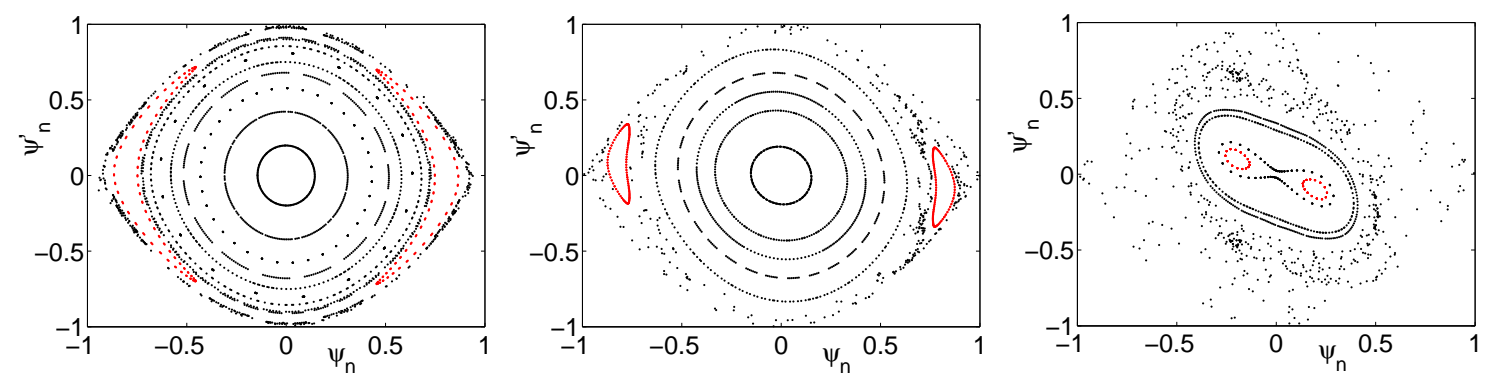

Figure 5. As figure 4, however for $\lambda=-0.02,-0.1,-0.5$ (from left to right).

The dynamics is quasiperiodic for small amplitudes, i.e. in the vicinity of the trivial solution $\left(\psi(x), \psi^{\prime}(x)\right)=(0,0)$. It is chaotic for large amplitudes at the edge of the snregion, corresponding to a stronger nonlinear interaction. The chaotic part of phase space becomes larger with increasing potential strength $|\lambda|$. The qualitative difference between stability and chaos is illustrated in Fig. 6, the 'dynamics' of the wave function $\psi(x)$ in configuration space and in phase space for $\mu=g=1$ and $\lambda=-0.2$ and $\lambda=-0.22$, respectively. In both cases, the initial state $\psi(0), \psi^{\prime}(0)$ was chosen as a periodic solution plus a small random perturbation (standard deviation $\sigma=10^{-3}$ ). This periodic solution is elliptically stable for $\lambda=-0.2$ so that the perturbation does not grow with $x$. It becomes hyperbolically unstable for $\lambda=-0.22$ so that the perturbation increases exponentially. Trajectories with different perturbations spread rapidly, as shown in Fig. 6 (c) and (d). The different forms of fixed points and their stability in dependence on the parameters will be discussed in detail in the next section.

As illustrated in Fig. 6 (c), a trajectory in the region of spatial chaos will generally diverge at some finite value of $x$. Such a trajectory is usually mapped close to the edge of the sn-region in phase space, where non-diverging solutions exist (cf. Fig. 1). Due to the discontinuity of the derivative $\psi^{\prime}(x)$ the wavefunction leaves the sn-region. As mentioned above, all non-sn-type solutions of the NLSE diverge at a finite value of $x$. Thus also trajectories started in the chaotic part of the sn-region usually diverge at a finite value of $x$. An example of such a diverging wavefunction is shown in Fig. 6] (c,d) for $\mu=g=1$ and $\lambda=-0.22$ (cf. Fig. 5). The initial value $\left(\psi(0), \psi^{\prime}(0)\right)$ was chosen as an unstable fixed point of $\tilde{f}$ inside the chaotic region plus a small random perturbation.

The stability or divergence of a wavefunction depends sensitively on the initial values $\left(\psi(0), \psi^{\prime}(0)\right)$. This is illustrated in Fig. 7 A grey-scale plot shows the point of divergence (the number of spatial periods until a trajectory diverges) in dependence of the initial condition $\left(\psi_{0}, \psi_{0}^{\prime}\right)$ for $\lambda=0.5$. The left figure shows the position of divergence for one quadrant of phase space, $\psi_{0}, \psi_{0}^{\prime} \geq 0$. This figure should be compared with the Poincaré section in Fig. 4. One clearly recognizes that trajectories with small amplitudes are quasiperiodic and thus stable. But one can also find initial values that do not lead to divergences for larger amplitudes. These values form an approximately self-similar set in phase space. This is illustrated in Fig. 7 in the lower plots, where magnifications of the upper plot are shown. 

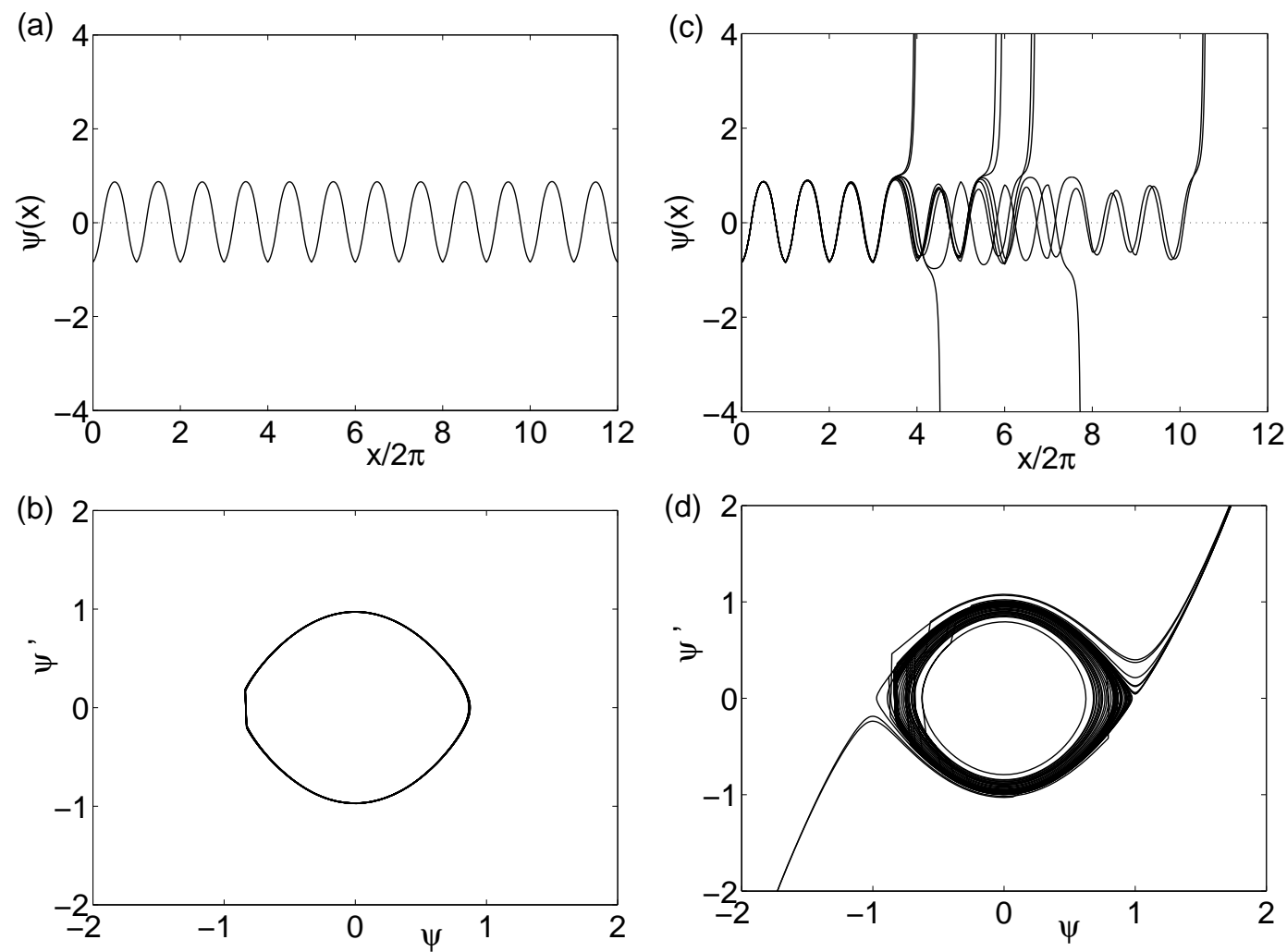

Figure 6. (In)stability of the wave function $\psi(x)$ for $\mu=g=1$ and $\lambda=-0.2(\mathrm{a}, \mathrm{b})$ and $\lambda=-0.22$ $(\mathrm{c}, \mathrm{d})$. The plots shows the 'dynamics' of the wave function $\psi(x)$ in configuration space (a,c) and in phase space (b,d). In all cases, the initial state $\psi(0), \psi^{\prime}(0)$ was chosen as the symmetric periodic solution plus a small random perturbation (standard deviation $\sigma=10^{-3}$ ). The periodic solution is elliptically for stable $\lambda=-0.2(\mathrm{a}, \mathrm{b})$ so that the error does not grow and the wave function $\psi(x)$ remains close to it for all $x$. The periodic solution becomes hyperbolically unstable for $\lambda=-0.22$ so that the error grows exponentially and the the wave function eventually diverges. Ten trajectories with different random perturbations are plotted in the figure, showing the rapid spreading of these trajectories.

\subsection{Attractive nonlinearity}

For the sake of completeness we also briefly discuss the case of an attractive nonlinearity $g<0$ without going into details.

Real solutions of the nonlinear Schrödinger equation for a delta comb can be constructed in terms of the Jacobi elliptic function $\mathrm{cn}$ and $\mathrm{dn}$. The cn-type solutions are given by

$$
\psi(x)=A_{n} \operatorname{cn}\left(4 K\left(p_{n}\right) \frac{x+x_{n}}{L_{n}} \mid p_{n}\right)
$$

for $x \in(2 \pi n, 2 \pi(n+1))$ with

$$
A_{n}^{2}=\frac{2 \mu p_{n}}{g\left(2 p_{n}-1\right)} \text { and } L_{n}^{2}=\frac{8\left(1-2 p_{n}\right) K\left(p_{n}\right)^{2}}{\mu} .
$$




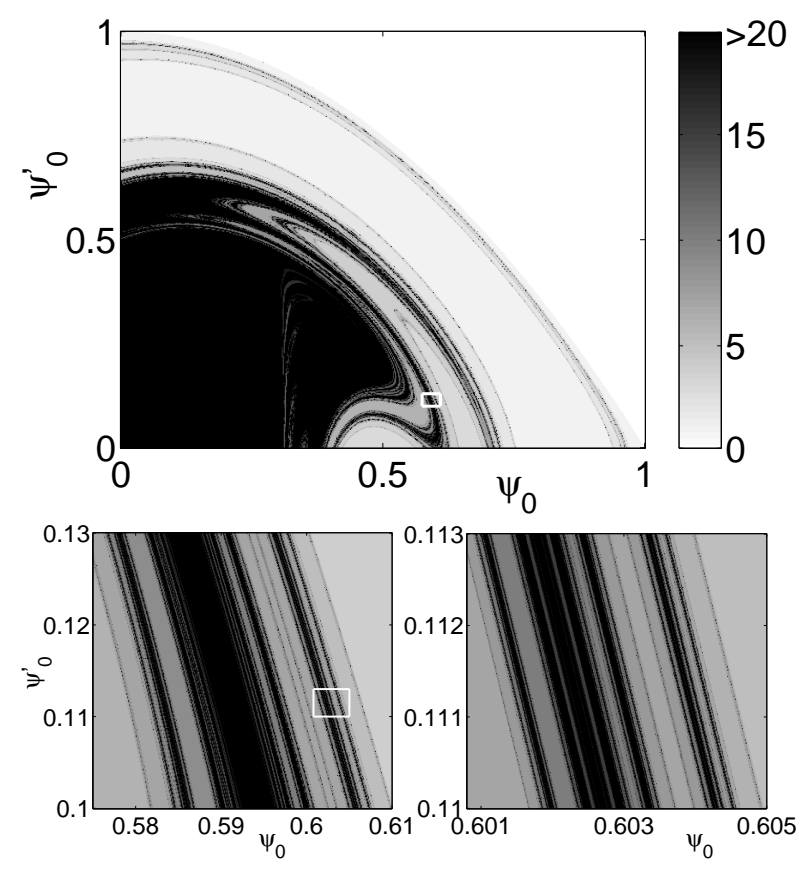

Figure 7. Number of spatial periods, at which a trajectory diverges in dependence of the initial condition $\left(\psi_{0}, \psi_{0}^{\prime}\right)$ for $\lambda=0.5$. The lower panels show subsequent magnifications.

The dn-type solutions read

$$
\psi(x)=A_{n} \operatorname{dn}\left(4 K\left(p_{n}\right) \frac{x+x_{n}}{L_{n}} \mid p_{n}\right)
$$

for $x \in(2 \pi n, 2 \pi(n+1))$ with

$$
A_{n}^{2}=\frac{\mu}{g(2-p)} \quad \text { and } \quad L_{n}^{2}=\frac{8(p-2) K(p)^{2}}{\mu} .
$$

Note that cn-type solutions exist for $\mu<0$, leading to $p \in(0.5,1]$, as well as for $\mu>0$, leading to $p \in[0,0.5)$, whereas the dn-type solutions exist only for $\mu<0$.

Again, two conditions have to be fulfilled at $x=2 \pi n$ : The wavefunction is continuous, whereas its derivative is discontinuous according to Eqn. (2.6). As above we can consider the dynamics stroboscopically at and thus arrive at the mapping $\tilde{f}:\left(\psi_{n}, \psi_{n}^{\prime}\right) \rightarrow$ $\left(\psi_{n+1}, \psi_{n+1}^{\prime}\right)$. Examples of the dynamics in phase space in a delta comb of strength $\lambda=-0.1$ are plotted in Fig. 8 .

An important difference to the case of a repulsive interaction is that one can find a solution of the free NLSE in terms of the non-diverging Jacobi elliptic functions cn and dn for all initial values of a wave function $\psi\left(x_{0}\right), \psi^{\prime}\left(x_{0}\right)$. This should be compared to the case of a repulsive interaction, in particular to Fig. 1. Hence the solutions for a delta-comb do not diverge at a finite value of $x$, regardless of the starting point $\left(\psi_{0}, \psi_{0}^{\prime}\right)$. The dynamics in phase space around $\left(\psi_{n}, \psi_{n}^{\prime}\right)=(0,0)$ is illustrated in Fig. 8 for $\lambda=-0.1$ and two different values of $\mu$. 

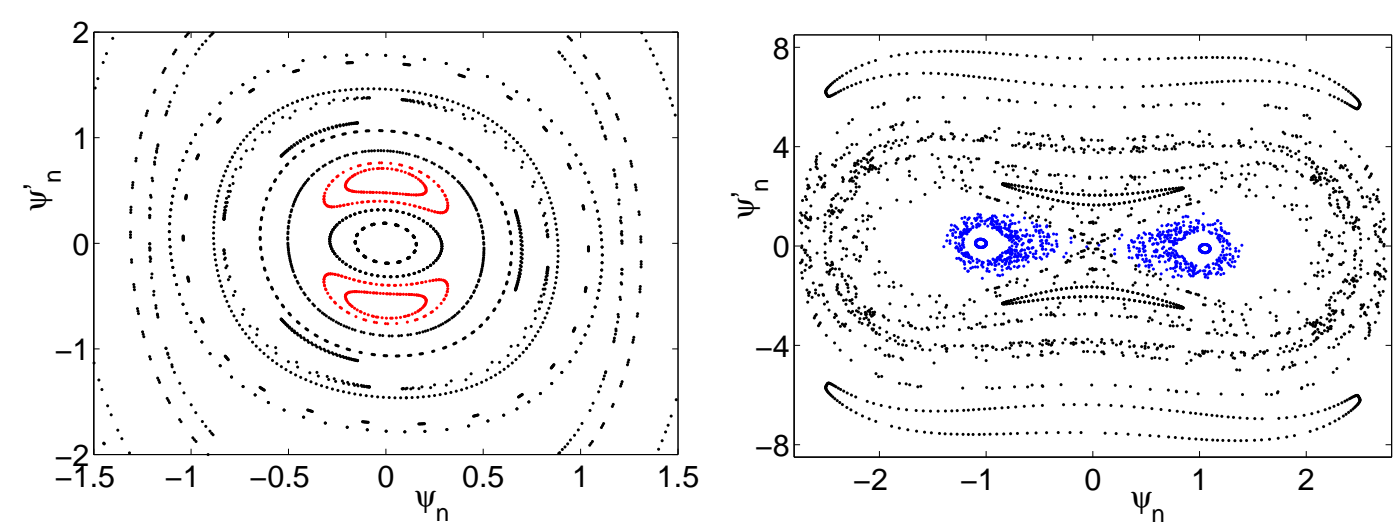

Figure 8. Stroboscopic phase space plots of the mapping $\tilde{f}:\left(\psi_{n}, \psi_{n}^{\prime}\right) \rightarrow\left(\psi_{n+1}, \psi_{n+1}^{\prime}\right)$ for an attractive nonlinearity $g=-1$ and $\mu=+1$ (left panel) and $\mu=-2$ (right panel).

The solutions are always given by the Jacobi elliptic function cn for $\mu>0$. For $\mu=1$ and $\lambda=-0.1$, the dynamics is still completely regular and one we can identify fixed points again (cf. the left panel of Fig. 8). The dynamics becomes partly chaotic for higher values of $\lambda$, but still a wave function cannot diverge at a finite value of $x$. However, a totally different mechanism of divergence exists in this case. The wave function $\psi(x)$ diverges for $x \rightarrow \infty$, if its phase is such that the changes of the amplitude due to the delta kicks accumulate. Revisiting Eqn. (2.17) one recognizes that this divergence of the amplitude $A_{n}$ implies $p_{n} \rightarrow 0.5$. Indeed one finds numerically that most trajectories in the chaotic region of phase space show such a behavior.

For $\mu<0$ the solution is described either by the function $c n$ or dn, depending on the initial value $\left(\psi_{0}, \psi_{0}^{\prime}\right)$. The dynamics in phase space is illustrated in the right panel of Fig. 8 for $\lambda=-0.1$. Solutions of dn-type are found around the elliptic fixed points at $\left(\psi_{n}, \psi_{n}^{\prime}\right)=( \pm 1,0)$. One can identify regular islands in a chaotic sea, surrounded by quasiperiodic orbits for large amplitudes. Again the cn-type solutions can diverge for $x \rightarrow \infty$ if $p_{n} \rightarrow 0.5$.

\subsection{Complex solutions}

Complex solutions of the free NLSE can be found by decomposing the wave function into amplitude and phase

$$
\psi(x)=\sqrt{S(x)} \mathrm{e}^{\mathrm{i} \phi(x)}
$$

with real-valued functions $\phi(x)$ and $S(x) \geq 0$. The phase is then given by

$$
\phi^{\prime}(x)=\frac{\alpha}{S(x)}
$$

with a real integration constant $\alpha$. In the limit $\alpha \rightarrow 0$ one recovers the real-valued snand cn-type solutions described above, which change sign at the zeros of the density. This limit is discussed in detail in [55] where interaction-induced transitions from scattering states to bound states are analyzed for a finite square well potential. 
We focus on the special solution $[11,55]$

$$
S(x)=B_{n}-\frac{\varrho_{n}^{2}}{g} \operatorname{dn}^{2}\left(\varrho_{n}\left(x+x_{n}\right) \mid p_{n}\right)
$$

of the free NLSE with $\varrho_{n}=4 K\left(p_{n}\right) / L_{n}$. Inserting this ansatz into the free NLSE yields the following conditions for the remaining constants

$$
\begin{aligned}
& 2 \mu=3 g B_{n}-\left(2-p_{n}\right) \varrho_{n}^{2} \\
& \varrho_{n}^{4}\left(p_{n}-1\right) B_{n}+g \alpha_{n}^{2}+2 g_{n}^{2} B_{n}-2 g \mu B_{n}^{2}=0 .
\end{aligned}
$$

For the delta-comb potential, one can make the ansatz (2.22) separately for each interval $x \in(2 \pi n, 2 \pi(n+1))$. The derivative $\psi^{\prime}$ of the the wave function is again discontinuous at $x=2 \pi n$ according to Eqn. (2.6). In terms of the density $S(x)$ this yields

$$
\lim _{\epsilon \rightarrow 0+}\left(S^{\prime}(2 \pi n+\epsilon)-S^{\prime}(2 \pi n-\epsilon)\right)=4 \lambda S(2 \pi n) .
$$

Complex periodic solutions of this type will be discussed in Sec. 3.3. These solutions are of great importance, since they occur for nonlinear Bloch bands (cf. Sec. 4).

\section{Periodic solutions}

Real periodic solutions can be viewed as fixed points of the mapping $f$ resp. $\tilde{f}$ defined in Sec. 2.2. In the following we calculate these solutions explicitly and discuss their spatial stability.

\subsection{Symmetric and antisymmetric real periodic solutions}

One kind of periodic solutions is found for $\psi(2 \pi n)=0$. They will be named antisymmetric periodic solutions in the following since they fulfill $\psi(2 \pi n-x)=-\psi(2 \pi n+x)$. In this case the wave function and its derivative are continuous everywhere. The period length of the Jacobi elliptic functions $L$ must fulfill

$$
L=4 \pi / m
$$

for $m \in \mathbb{N}$. The antisymmetric solutions are $2 \pi$-periodic only for even $m$, since their fundamental period is $4 \pi / \mathrm{m}$. Note that $m$ is the number of nodes of the wave function in $[0,2 \pi)$. In the following we will mostly consider the "primary resonance" $m=2$ with the fundamental period $2 \pi$. However, we will show in Sec. 3.2 that this solution bifurcates when $\lambda$ is varied and new period doubled solutions emerge.

In terms of the elliptic parameter $p$ Eqn. (3.1) reads

$$
(p+1) K(p)^{2}=\frac{2 \pi^{2} \mu}{m^{2}} .
$$

The left side of this equation is bounded from below by $(p+1) K(p)^{2} \geq K(0)^{2}=\pi^{2} / 4$. Thus antisymmetric periodic solutions only exist if $\mu \geq m^{2} / 8$. Comparing with the case of 

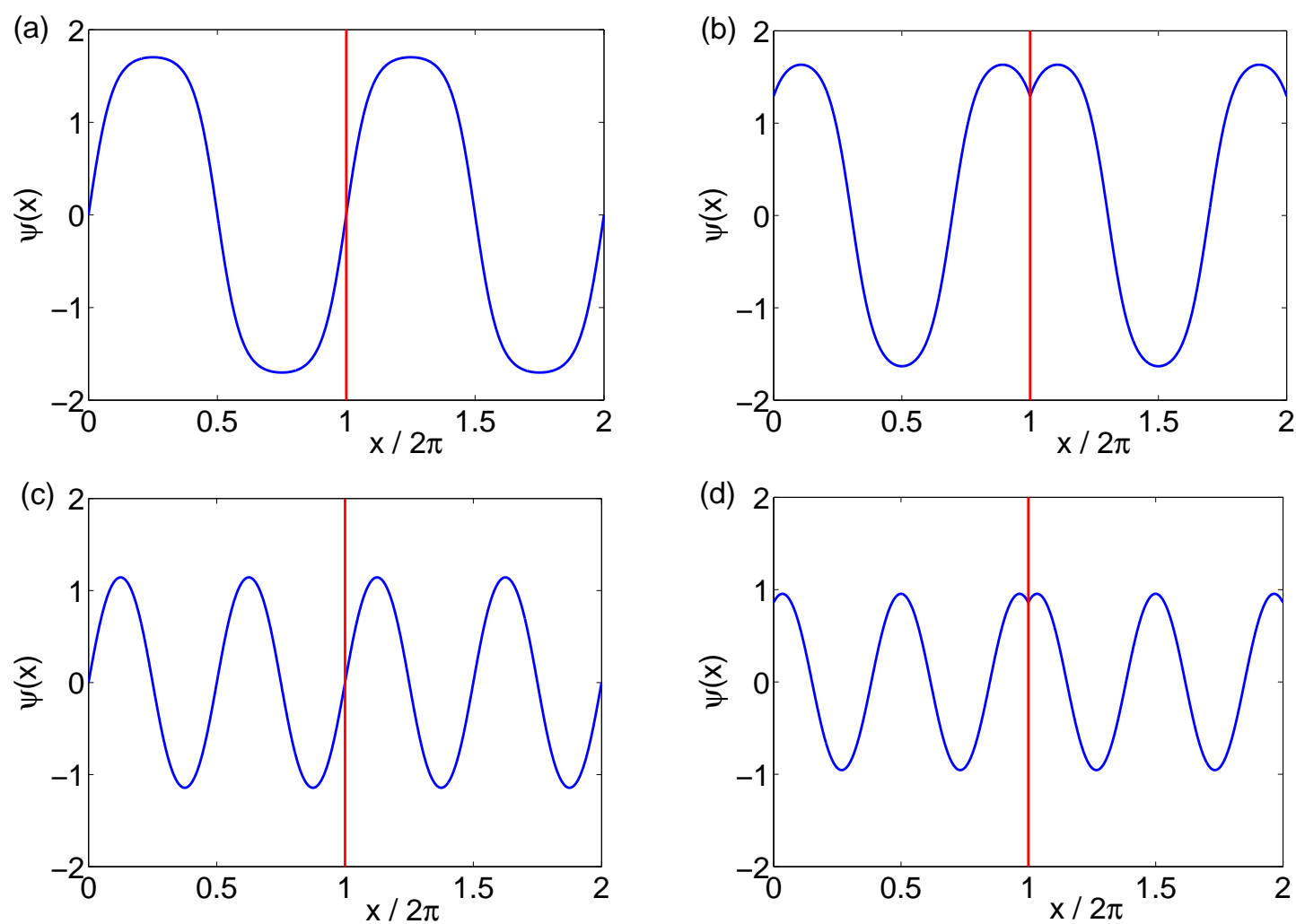

Figure 9. Periodic solutions $\psi(x)$ of the NLSE for a delta-comb potential of strength $\lambda=1$ for $\mu=3$ and $g=1$. The red vertical lines indicate the positions of the delta potentials. Antisymmetric solutions (a,c) have a node at the positions of the delta potential, $\psi(2 \pi n)=0$, so that they experience no discontinuity of the first derivative and are thus independent of $\lambda$. Symmetric solutions $(\mathrm{b}, \mathrm{d})$ are non-zero at $x=2 \pi n$ and symmetric with respect to a reflexion at this point. Furthermore the solutions are characterized by the number of nodes in the interval $[0,2 \pi)$ - the examples have $m=2(\mathrm{a}, \mathrm{b})$ and $m=4(\mathrm{c}, \mathrm{d})$ nodes, respectively.

a harmonic potential, i.e. the nonlinear Mathieu equation [43,48], one recognizes that an antisymmetric periodic solution comes into being just at the $2: m$ resonance $2 \mu=m^{2} / 4$.

The shift $x_{n}$ is given by $x_{n}=0$ resp. $x_{n}=\pi$. However, these solutions differ only by an overall sign. Note that the solution of Eqn. (3.2) does not depend on $\lambda$. As an example the antisymmetric periodic solutions with $m=2$ and $m=4$ re shown in Fig. 9 (a) and (c), respectively, for $\mu=3$ and $g=1$.

Furthermore, $2 \pi$-periodic solutions can be found which are symmetric around the positions of the delta potentials at $x=2 \pi n, n \in \mathbb{Z}$. This symmetry and the periodicity of the wave function imply that the solution is also symmetric around $x=(2 n+1) \pi$, as one can easily see:

$$
\psi((2 n+1) \pi-x)=\psi(-(2 n+1) \pi+x)=\psi((2 n+1) \pi+x)
$$

Hence the the wave function assumes a maximum or minimum at $x=(2 n+1) \pi$, in the 
middle between two delta potentials. Without loss of generality we conclude that

$$
x_{n}=-(2 n+1) \pi+L / 4 \quad \text { or } \quad x_{n}=(2 n-1) \pi+3 L / 4 .
$$

These solutions differ only by an overall sign, hence only one of them must be considered. Again condition (2.6) must be fulfilled at the positions of the delta-comb. Because of the periodicity it is, however, sufficient to evaluate this equation at $x=0$ only. A further simplification arises from the symmetry of the wave function, yielding the condition

$$
\lambda \operatorname{sn}(u \mid p)=\frac{4 K(p)}{L} \operatorname{cn}(u \mid p) \operatorname{dn}(u \mid p)
$$

with $u=4 K(p) x_{0} / L=K(p)(1-4 \pi / L)$. Eqn. (3.5) has a solution for every $\mu>0$. For higher values of $\mu$ it may have several solutions, differing in the period $L$ and correspondingly in the number of nodes of the wave function. Again, these solutions will be labeled by the number $m$ of nodes of the wave function in the interval $[0,2 \pi)$. In the following we consider the solution with $m=2$ nodes. Exemplarily the solutions with $m=2$ and $m=4$ are shown in Fig. 9 (b) and (d), respectively, for $\mu=3, g=1$ and $\lambda=1$.

One can easily see that these two solution classes are the only possible $2 \pi$-periodic sn-type wave functions. Due to the periodicity the elliptic parameter $p_{n}$ is the same for all $n$, i.e. $p_{n}=p$. As the elliptic parameter $p$ is fixed, the derivative $\psi^{\prime}\left(x_{0}\right)$ at a point $x_{0}$ is determined by the wave function $\psi\left(x_{0}\right)$ up to a sign. Considering $x_{0}=2 \pi n$ this leaves the possibilities $\psi^{\prime}(2 \pi n+\epsilon)=\psi^{\prime}(2 \pi n-\epsilon)$, for which one recovers the antisymmetric periodic solutions, whereas the other possibility $\psi^{\prime}(2 \pi n+\epsilon)=-\psi^{\prime}(2 \pi n-\epsilon)$ leads to the symmetric periodic solutions.

\subsection{Spatial Stability of the real periodic solutions}

As already stated, real periodic solutions of the NLSE in a delta-comb are the fixed points of the mapping $f$ resp. $\tilde{f}$, defined in Sec. 2.2. Similarly, we can find solutions with a periodicity of $2 \pi r$ as fixed points of the mapping $f^{r}$. In the following we discuss the characteristics of these fixed points, especially their spatial stability, in dependence of the control-parameter $\lambda$.

First we discuss the antisymmetric periodic solutions with $L=2 \pi / m$ for a repulsive nonlinearity $g=1$ and $\mu=1$. As an example, we consider the solutions with $m=2$, which have nodes at $x=\pi n, n \in \mathbb{Z}$, i.e. at the positions of the delta-potentials $x=2 \pi n$ and additionally in the middle between them. These periodic solutions are found for $p \approx 0.4719$ and $x_{n}=0$, resp. $x_{n}=\pi$, differing only by an overall sign.

To determine the stability of a fixed point numerically we iterate the mapping $f$, where the initial value $\left(p_{0}, x_{0}\right)$ is chosen as the fixed point plus a small random perturbation. Furthermore we calculate the stability (or monodromy) matrix of the mapping $\tilde{f}$

$$
M\left(\psi_{*}, \psi_{*}^{\prime}\right)=\left[\frac{\partial\left(\psi_{n+1}, \psi_{n+1}^{\prime}\right)}{\partial\left(\psi_{n}, \psi_{n}^{\prime}\right)}\right]_{\psi_{*}, \psi_{*}^{\prime}}
$$

at the fixed point $\left(\psi_{*}, \psi_{*}^{\prime}\right)$. As the mapping $\tilde{f}$ is area-preserving, the product of the eigenvalues $\exp ( \pm \gamma)$ of $M$ is unity. The mapping is unstable if the stability exponent $\gamma$ is 

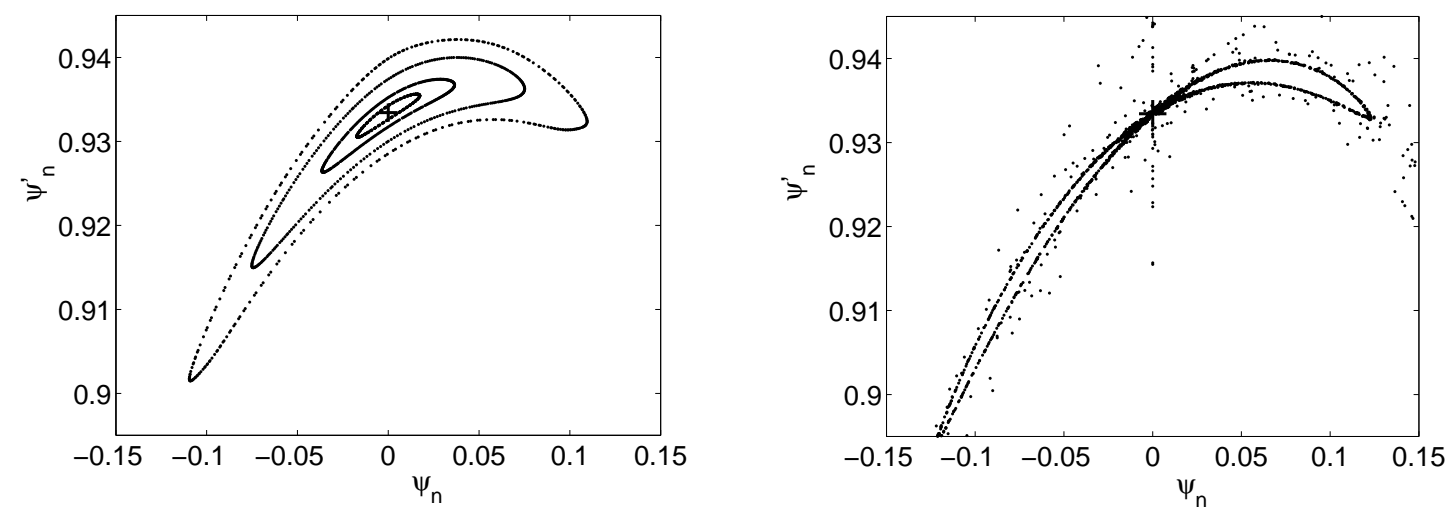

Figure 10. Phase space around the antisymmetric fixed point of $f$ (marked by a + ) before the bifurcation $(\lambda=0.14$, left $)$ and after the bifurcation $(\lambda=0.16$, right $)$.
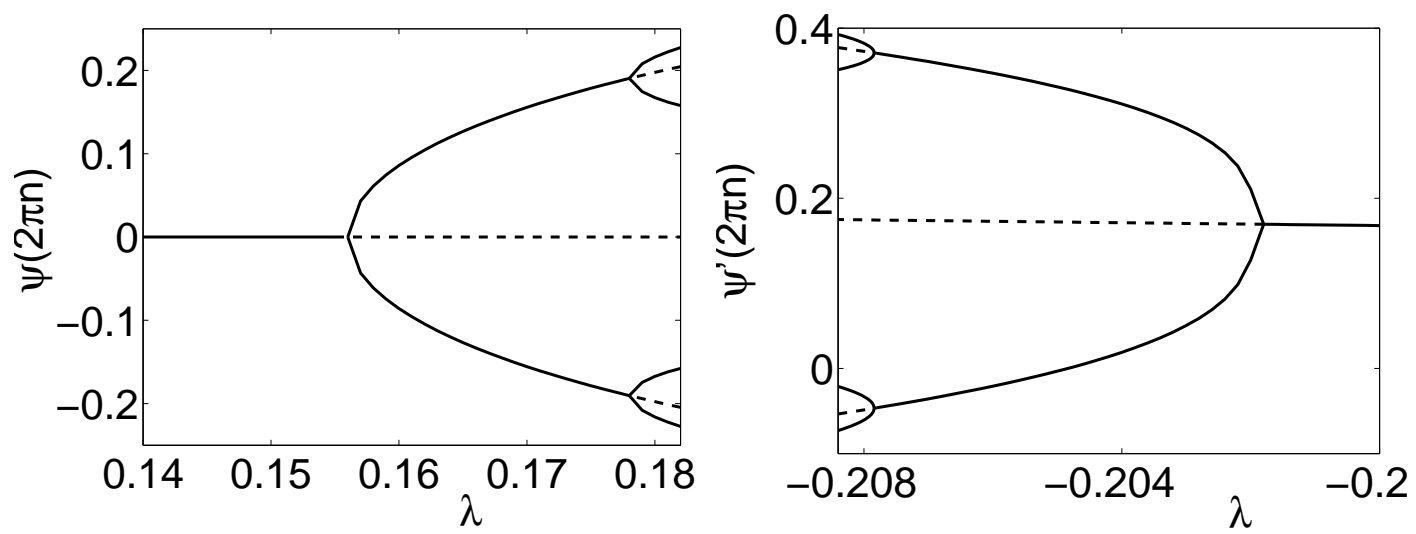

Figure 11. Period doubling of the antisymmetric fixed point with $m=2$ (left panel) and of the symmetric fixed point (right panel) for $\mu=g=1$. The Fixed points of $f, f^{2}$ and $f^{4}$ are plotted as a function of the control-parameter $\lambda$. Stable fixed points are indicated by a solid line, unstable fixed points by a dashed line.

real. Otherwise it is purely imaginary and the mapping is elliptically stable. Then $|\gamma|$ is called the stability angle.

Exemplarily we consider the antisymmetric fixed point with $m=2$, which is found to be elliptically stable if $\lambda>0$ and $\lambda$ is not too large. The stability exponents $\gamma_{ \pm}$are purely imaginary and one finds quasiperiodic orbits around the fixed point. This is shown in the left panel of Fig. 10 for $\lambda=0.14$. The fixed point becomes unstable when the control-parameter $\lambda$ is increased above a critical value $\lambda_{c} \approx 0.156$. This is illustrated in the right panel of Fig. 10 for $\lambda=0.16$.

In fact, a bifurcation occurs at the critical value $\lambda_{c}$ and two new fixed points of $f^{2}$ emerge. This is illustrated in Fig. 11, where $\psi_{n}=\psi(2 \pi n)$ is plotted for the fixed points of $f^{r}, r=1,2,4$ as a function of $\lambda$. One clearly observes a pitchfork bifurcation scenario. The new fixed point of $f^{2}$ no longer fulfills $\psi(2 \pi n)=0$, but instead $\psi(2 \pi n)=-\psi(2 \pi(n+1))$. A second bifurcation occurs at $\lambda_{c}^{\prime} \approx 0.178$, where the fixed points of $f^{2}$ become unstable 


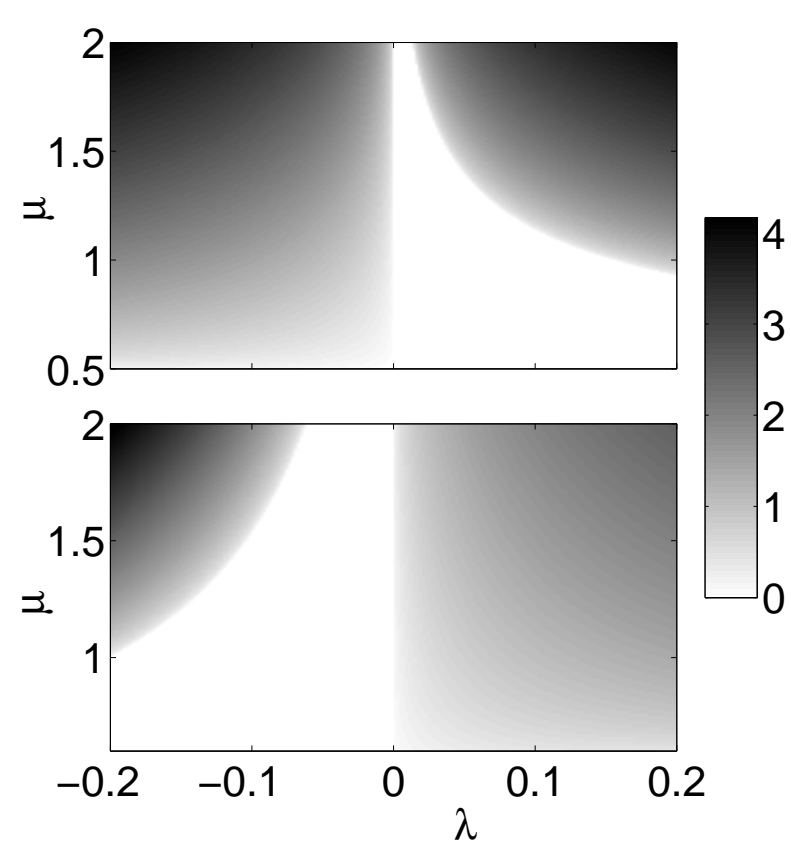

Figure 12. Stability exponent $\operatorname{Re}(\gamma)$ for the antisymmetric fixed point with $m=2$ (two nodes in $[0,2 \pi)$, upper panel) and the symmetric fixed point (lower panel) as a function of $\mu$ and $\lambda$ in a greyscale plot. Wave functions corresponding to these fixed points are illustrated in Fig. 9. Stable regions are colored in white $(\operatorname{Re}(\gamma)=0)$.

and four new elliptically stable fixed points of $f^{4}$ emerge. These fixed points again become unstable at $\lambda_{c}^{\prime \prime} \approx 0.182$.

One should keep in mind that the NLSE represents a Hamiltonian system and that the period doubling scenario of Hamiltonian systems (cf. [3,42]) is different from the familiar Feigenbaum scenario for dissipative systems. For instance, the stable fixed points are always elliptically (resp. neutrally) stable and not asymptotically stable.

For $\lambda<0$ the antisymmetric fixed points are unstable. However, large regular regions exist in phase space around the trivial solution $\psi(x) \equiv 0$ and the symmetric fixed points as long as $|\lambda|$ is not too large (cf. Fig. 55). A "trajectory" started in the vicinity of an antisymmetric fixed point is unstable but it may get trapped in the chaotic sea between the large regular regions and consequently show quasi-regular dynamics over many periods. For stronger potentials, i.e. larger values of $|\lambda|$, the regular regions shrink and cannot trap such a trajectory any longer so that it will in general diverge.

A similar stability behavior is found for the symmetric fixed point. However, this solution is always unstable for a repulsive delta-comb, $\lambda>0$, and shows a bifurcation scenario for an attractive delta-comb, $\lambda<0$. For small repulsive potentials, a similar trapping scenario occurs as described above for the antisymmetric fixed points in an attractive delta-comb. Again this trapping is lost for stronger potentials, since the regular regions of phase space shrink and the trajectories finally diverge in general.

The symmetric fixed points are elliptically stable for an attractive delta-comb as long as $\lambda>\lambda_{c}$. A bifurcation occurs if $\lambda$ is decreased below the critical value $\lambda_{c} \approx-0.203$. The $4 \pi$-periodic states are again elliptically stable up to the next bifurcation. This bifurcation 


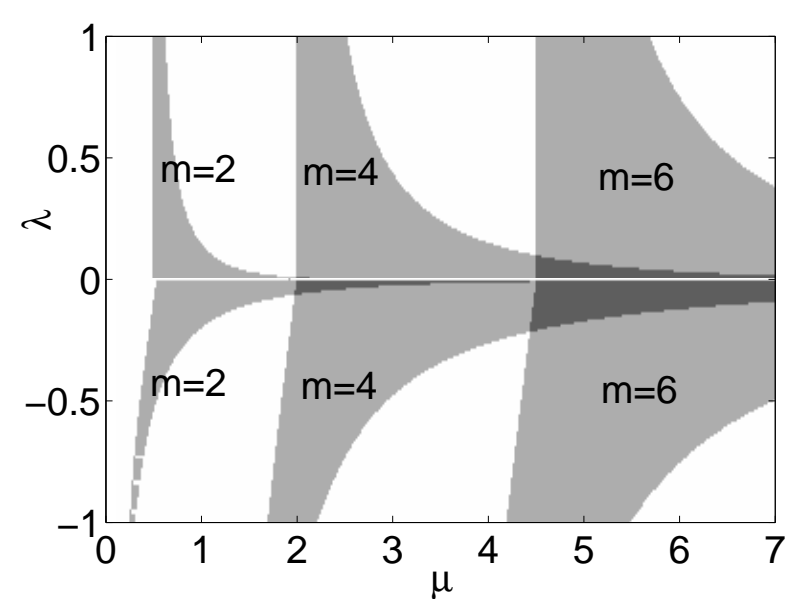

Figure 13. Stability map of the periodic states for a delta-comb with $g=1$. The periodic solutions are stable in the shaded areas of the parameter plane (antisymmetric states for $\lambda>0$ and symmetric states for $\lambda<0$ ). The different solutions are labeled by the number of nodes $m$ in the interval $[0,2 \pi)$.

route is illustrated in Fig. 11 for the symmetric periodic solution with $m=2$ nodes in the interval $\mid 0,2 \pi)$.

Note that also the trivial solution $\psi(x) \equiv 0$ of the NLSE shows a bifurcation when $\lambda$ is decreased. The trivial solution is stable for $\lambda \geq \lambda_{c}^{(0)} \approx-0.391$, where two new elliptically stable period doubled fixed points emerge. Quasiperiodic orbits around the emerging fixed points are illustrated in the lower panel of Fig. 5 for $\lambda=-0.5$.

The stability of the antisymmetric and the symmetric fixed point with $m=2$ is summarized in Fig. 12, where the stability exponent $\operatorname{Re}(\gamma)$ is plotted as a function of $\mu$ and $\lambda$. Fig. 13 shows a stability map of several fixed points with different $m$.

One can easily understand the qualitative differences of the stability between $\lambda<0$ and $\lambda>0$ by considering the full phase-space dynamics of the classical analogon (2.1). An antisymmetric periodic solution is not affected by the delta-comb because of $\psi(2 \pi n)=0$. However, a trajectory with a slightly larger classical energy moves ahead of the periodic solution, hence it experiences a kick. This kick will increase the energy of the classical oscillator if $\lambda>0$. The effect of the kicks accumulate and consequently the trajectory becomes unstable. If $\lambda<0$ the kick will lower the energy and stabilize the trajectory. The contrary effect occurs for the symmetric periodic solution. However, the situation is a bit more involved here since the periodic solution itself experiences the delta-kicks.

\subsection{Complex periodic solution}

Complex periodic solutions can be found using the ansatz (2.22). By similar arguments as above one finds that the density $S(x)=|\psi(x)|^{2}$ must be symmetric around $x=2 \pi n$. Hence it must assume a maximum or minimum at $x=(2 n+1) \pi$, in the middle between two delta-peaks. Therefore the "phase shift" $x_{n}$ is given by

$$
x_{n}=-(2 n+1) \pi \quad \text { or } \quad x_{n}=-(2 n+1) \pi+L / 4 .
$$


Using the symmetry of the density, the continuity of Eqn. (2.23) yields the condition

$$
\frac{\varrho^{2} p}{g \lambda} \operatorname{sn}(u \mid p) \operatorname{cn}(u \mid p) \operatorname{dn}(u \mid p)=B-\frac{\varrho^{2}}{g} \operatorname{dn}^{2}(u \mid p)
$$

with $u=4 K(p) x_{n} / L$ for a periodic complex solution. An example of such a periodic solution is shown in Fig. 15 (dashed line).

For $\alpha \rightarrow 0$ the complex solutions tend to the real-valued sn-type solutions which give rise to antisymmetric periodic solutions as discussed above. In fact, this is exactly what happens at the occurrence of loop structures in nonlinear Bloch bands at $\kappa=0$ (cf. Sec. 4).

\section{Nonlinear Bloch bands}

Recently, nonlinear Bloch states and Bloch bands have attracted considerable attention. New features such as looped Bloch bands [65,69,71] and period doubled Bloch states [40] were found. Nonlinear Bloch bands for the delta-comb were first calculated by Seaman et al. [57]. In this section we want to link these results to the properties of periodic solutions discussed in Sec. 3 .

The appearance of looped bands, or more generally the bifurcation of eigenvalues in nonlinear systems, is intimately related to branch point singularities, i.e. exceptional points (see [14] and references therein). Those exceptional points are eigenvalue and eigenvector degeneracies, which for linear systems can only appear in a non-hermitian description. As a boundary of regions of purely real eigenvalues they appear in so called PT-symmetric models. In this context, bifurcation phenomena of Bloch bands are also discussed in linear, however non-hermitian periodic potentials with PT-symmetry [17, 18,41]. A full understanding of the interrelation of these phenomena has not yet been achieved and may be provided by an analysis of the full nonlinear and non-hermitian periodic potential in an extension of the analysis of the corresponding two-state case (see, e.g., $[28,66]$ ). This is, however, only in the beginning (see [46] for a very recent study).

Looped Bloch bands arise in nonlinear systems when a space-periodic stationary solution of the NLSE (a Bloch state with quasimomentum $\kappa=0$ ) undergoes a bifurcation associated with the emergence of a dynamical instability. Before the bifurcation, this Bloch state is neutrally stable with respect to small perturbations in the sense that the time-dependent wave function $\psi(x, t)$ will remain close to the initial state $\psi(x, t=0)$.

In the bifurcation, two novel neutrally stable Bloch states emerge, while the old state becomes hyperbolically unstable in the the sense that the time-dependent wave function $\psi(x, t)$ rapidly spreads from the the initial state $\psi(x, t=0)$ leading to full spatio-temporal chaos, an example of which is shown in Fig. 22. Such a dynamical instability leads to a divergence of the Bose-Einstein condensate mode so that a simple mean-field approximation is no longer valid. Nevertheless, the time-dependent NLSE is still meaningful since it accurately predicts the occurence of the instability and the rate of the depletion $[15,16,62,63]$.

On the contrary, period-doubled Bloch bands come into existence when a usual $2 \pi$ periodic solution $\psi(x)$ of the NLSE undergoes a period-doubling bifurcation to form a $4 \pi$-periodic solution. Consequently, the $2 \pi$-periodic wave function is spatially stable up to the bifurcation where it becomes spatially unstable as illustrated in Fig. 6. 


\subsection{Linear and nonlinear Bloch states}

Bloch states are nonlinear eigenstates of the form

$$
\psi_{\kappa}(x)=\mathrm{e}^{\mathrm{i} \kappa x} u_{\kappa}(x),
$$

where $u_{\kappa}(x)$ is $2 \pi$-periodic and $\kappa$ denotes the quasimomentum. The periodic functions $u_{\kappa}(x)$ fulfill the differential equation

$$
-\frac{1}{2}\left(\frac{\mathrm{d}}{\mathrm{d} x}+\mathrm{i} \kappa\right)^{2} u_{\kappa}(x)+V(x) u_{\kappa}(x)+g\left|u_{\kappa}(x)\right|^{2} u_{\kappa}(x)=\mu(\kappa) u_{\kappa}(x)
$$

The eigenenergies $\mu(\kappa)$ form the nonlinear Bloch bands. In the preceding sections we analyzed the solutions of the NLSE in dependence of the parameter $\mu$, whereas the normalization of the wave function was arbitrary. For example, periodic states, which are Bloch states for $\kappa=0$, exist for any value of $\mu>0$ (cf. Sec. 3.1). Considering $\mu$ as a parameter is of course not appropriate here, since this would lead to a continuous and multiply degenerate spectrum of $\mu(\kappa)$ for every $\kappa$, whereas the corresponding Bloch states differ by their normalization. Consequently we consider $2 \pi N$-periodic Bloch states here, whose normalization is fixed as

$$
\frac{1}{2 \pi N} \int_{0}^{2 \pi N}\left|u_{\kappa}(x)\right|^{2} \mathrm{~d} x=1
$$

throughout this section, leading to a discrete Bloch spectrum. The Bloch states and the bands are calculated as described in [71].

The set of Bloch states is characterized by one continuous parameter, the quasimomentum $\kappa$, and an integer number counting the bands. General non-diverging stationary states are given by four continuous parameters, the real and imaginary parts of the initial value $\left(\psi_{0}, \psi_{0}^{\prime}\right)$. Thus the Bloch states are of measure zero within the the set of all possible non-diverging stationary states. The nonlinear stationary states in a periodic potential generally do not fulfill Bloch's theorem.

\subsection{Looped Bloch bands}

The nonlinear Bloch bands for a delta-comb potential $V(x)=\lambda \delta_{2 \pi}(x)$ are shown in Fig. 14 for $g=1 / \pi$ and $\lambda=0.5$. The left-hand side shows the lowest bands in comparison to the linear case $g=0$, whereas the right hand side illustrates the emergence of loop structures at the upper edge of the lowest band at $\kappa=0.5$. Similarly loops emerge at the edge of the first excited band at $\kappa=0$. The chemical potential is larger than in the linear case by an amount of approximately

$$
\frac{g}{2 \pi} \int_{0}^{2 \pi N}\left|u_{\kappa}(x)\right|^{4} \mathrm{~d} x
$$

because of the repulsive mean-field potential. Considering the energy per particle instead of the chemical potential one finds swallow's tail structures instead of the loops $[57,65]$.

The Bloch states with $\kappa=0$ are strictly periodic and can be chosen real up to the emergence of loops at a critical nonlinearity $g_{\text {loop }}$. These states can be identified with the 

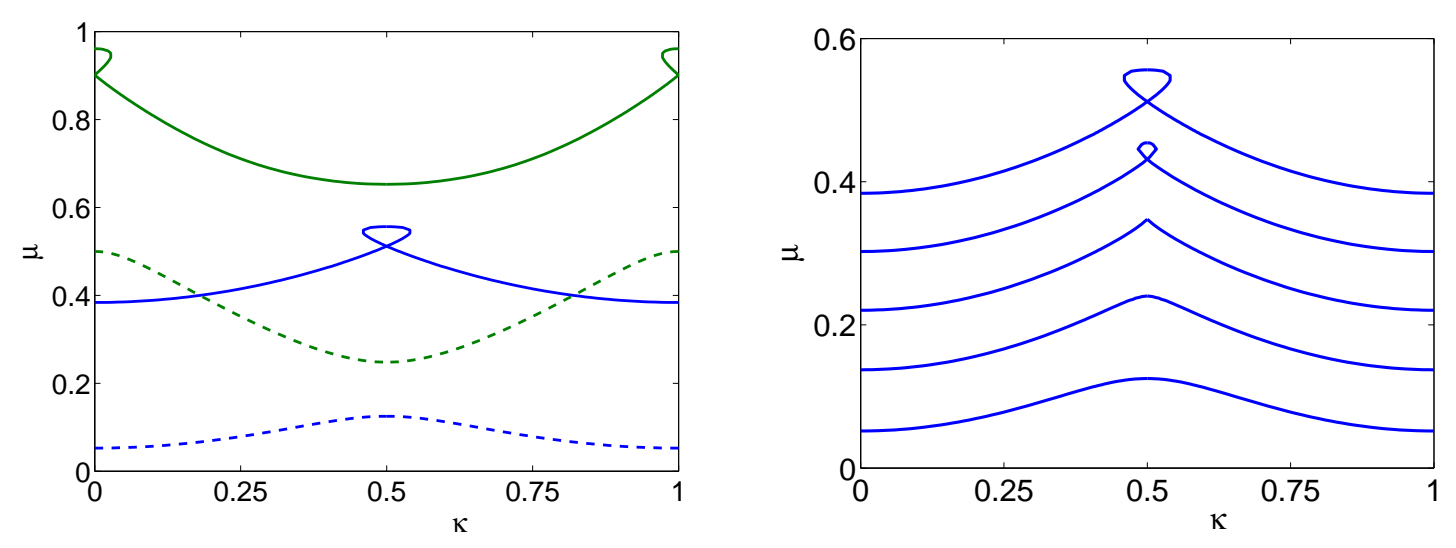

Figure 14. Nonlinear Bloch bands and emergence of loop structures for a delta-comb potential. Left: The lowest nonlinear Bloch bands for $g=1 / \pi$ (solid lines) in comparison to the linear case $g=0$ (dashed lines). Right: The lowest Bloch band for $2 \pi g=0,0.5,1,1.5,2$ (from bottom to top) and $\lambda=0.5$.

symmetric resp. antisymmetric periodic states described in Sec. 3.1. It is found that the Bloch state in the lowest band is a symmetric periodic solution with no node, while the state in the first excited band is an antisymmetric periodic solution with $m=2$ nodes in the interval $[0,2 \pi)$.

Loops emerge for $g>g_{\text {loop }}$, hence additional Bloch states with $\kappa=0$ come into being. A state corresponding to the self-crossing of a looped band at $\kappa=0$ cannot be chosen purely real, whereas the state at the top of the loop can. It is found that the state at the top of the loop in the first excited band still corresponds to a real antisymmetric periodic solution as for $g<g_{\text {loop. }}$. The Bloch state at the self-crossing corresponds to a complex periodic solution as introduced in Sec. 3.3. The Bloch states in the two lowest bands at $\kappa=0$ are shown in Fig. 15 for $g=1 / \pi$ and $\lambda=0.5$. The squared modulus of a symmetric periodic solution (ground band) and the antisymmetric periodic solution with $m=2$ (first excited band) are plotted as a bold (symmetric) resp. solid line (antisymmetric). The complex solutions that degenerate at the self-crossing at $\kappa=0$ transform into each other by a sign change of $\kappa$ and consequently by a sign change of $x$ (cf. Eqn. (4.2)). The squared modulus of these two solutions is the same and hence symmetric around $x=0$ (dashed line in Fig. 15), whereas the phases are antisymmetric: $\phi(-x)=-\phi(x)$.

Using the explicit form of the real and complex periodic solutions one can derive the critical nonlinearity $g_{\text {loop }}$ for the emergence of loops in the first excited Bloch band. To this end we reconsider condition (3.8) for the complex periodic solutions. For $g \rightarrow g_{\text {loop }}$ the complex periodic solution and the real antisymmetric solution merge. In this limit the wave function tends to zero at the positions of the delta-peaks, $\psi(2 \pi n) \rightarrow 0$, and its derivative becomes continuous. Hence both sides of Eqn. (3.8) tend to zero.

Consider a complex periodic solution slightly above the critical point. The "wave number" $\varrho$ is written as $\varrho=\varrho_{\text {loop }}+\delta \varrho$, where $\varrho_{\text {loop }}=m K(p) / \pi$ is the wave number at 

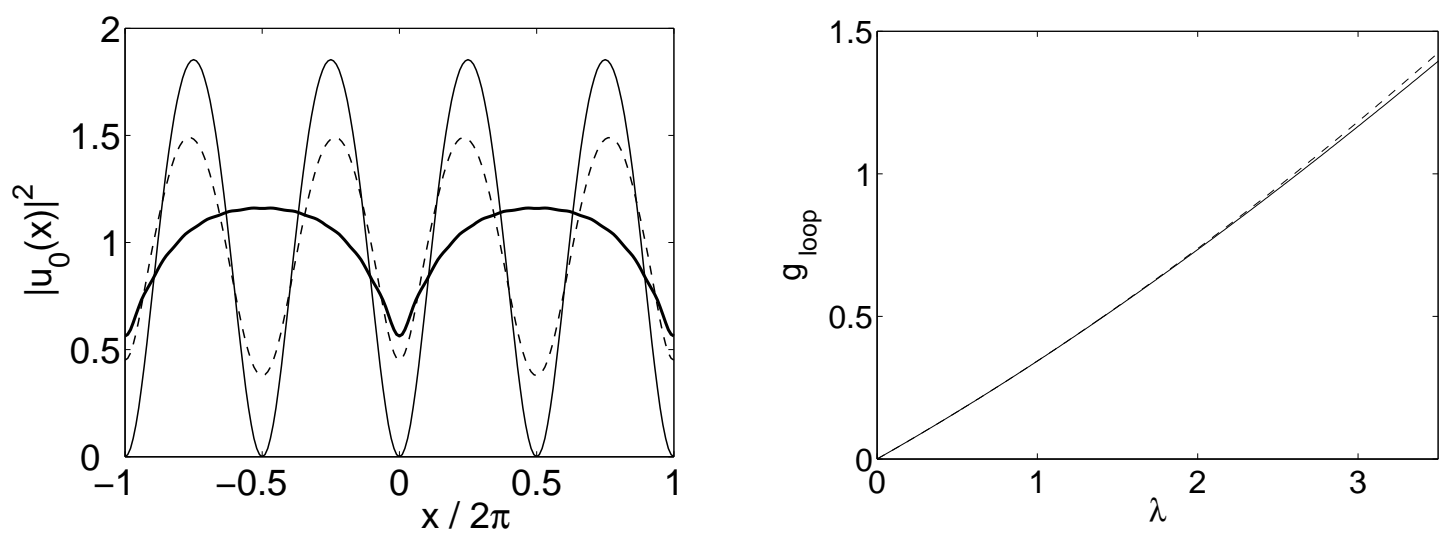

Figure 15. Left: Squared modulus of the wave function of nonlinear Bloch states for $\kappa=0$, $g=1 / \pi$ and $\lambda=0.5$ (bold line: lowest band, dashed line: state at the self-crossing of the loop in the first excited band, thin solid line: state at the top of the loop). Right: Dependence of the critical nonlinearity $g_{\text {loop }}$ for the emergence of loops in dependence of the potential strength $\lambda$ (solid line) and the approximation (4.9) (dashed line).

the critical point $g=g_{\text {loop }}$. Expanding Eqn. (3.8) around $\varrho_{\text {loop }}$ with fixed $B$ then yields

$$
-\frac{2 p \varrho_{\text {loop }}^{3}}{g} \pi \delta \varrho+\mathcal{O}\left(\delta \varrho^{2}\right)=\underbrace{2 \lambda B-\frac{2 \lambda \varrho_{\text {loop }}^{2}}{g}}_{=0}-\frac{4 \lambda \varrho \text { loop }}{g} \delta \varrho+\mathcal{O}\left(\delta \varrho^{2}\right) \text {. }
$$

Comparing the coefficients of the linear term yields an equation for the elliptic parameter $p_{\text {loop }}$ at the critical point

$$
p_{\text {loop }} K\left(p_{\text {loop }}\right)^{2}=\frac{2 \pi \lambda}{m^{2}} .
$$

The normalization of the wave function at the critical point yields

$$
2 \pi \stackrel{!}{=} \int_{0}^{2 \pi} A^{2} \operatorname{sn}^{2}\left(4 K(p) \frac{x+x_{n}}{L} \mid p\right)=\frac{2 m^{2}}{\pi g} K(p)[K(p)-E(p)],
$$

where $K(p)$ and $E(p)$ denote the complete elliptic integrals of the first and second kind, respectively. Hence the critical nonlinearity is given by

$$
g_{\text {loop }}=\frac{m^{2}}{\pi^{2}} K\left(p_{\text {loop }}\right)\left[K\left(p_{\text {loop }}\right)-E\left(p_{\text {loop }}\right)\right],
$$

where $p_{\text {loop }}$ is determined by Eqn. (4.6). One can derive an approximate explicit expression for the critical nonlinearity parameter $g_{\text {loop }}$ by expanding the left-hand side of Eqn. (4.6) up to second order in $p_{\text {loop }}$ and substituting the result into Eqn. (4.8). This yields in second order in $p_{\text {loop }}$

$$
g_{\text {loop }} \approx \frac{\lambda}{\pi}+\frac{\lambda^{2}}{\pi^{2} m^{2}}
$$

The dependence of $g_{\text {loop }}$ on $\lambda$ is shown in the lower panel of Fig. 15] for $m=2$, which corresponds to the first excited Bloch band. 

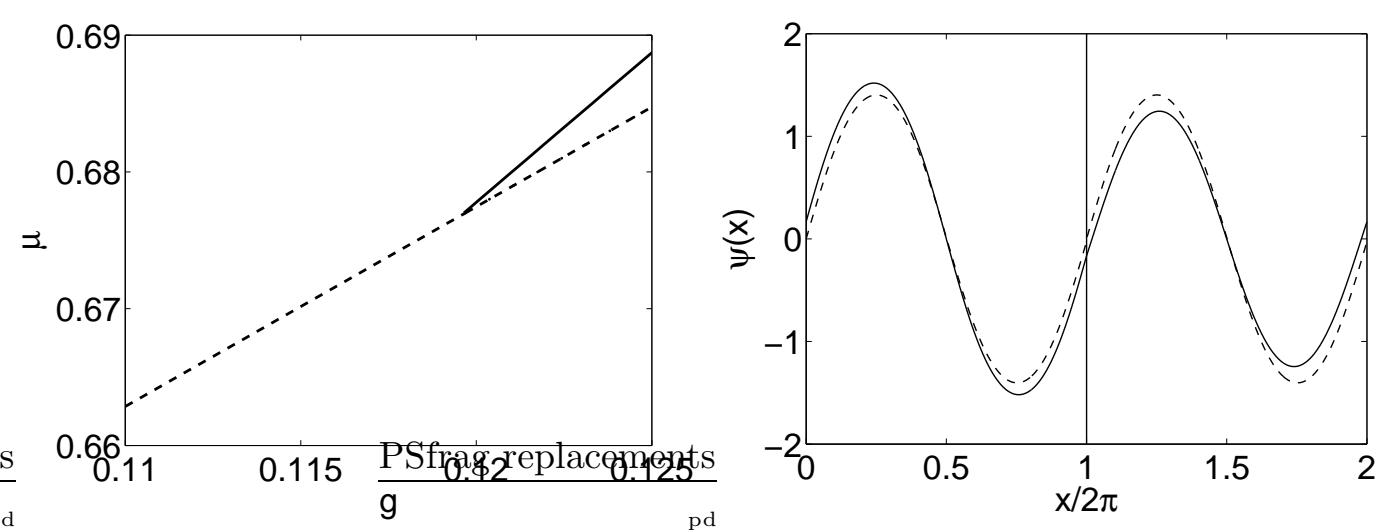

Figure 16. Left panel: Bifurcation of the $2 \pi$-periodic state corresponding to the first excited Bloch band by a variation of $g$. Shown is the chemical potential $\mu$ of the $2 \pi$-periodic state $(---)$ and the emerging $4 \pi$-periodic state $(-)$. Right: Wave function of the $2 \pi$-periodic $(---)$ and the $4 \pi$-periodic (-) state for $g=1 / 8$.

\subsection{Period doubled Bloch bands}

Let us finally discuss another new feature of nonlinear Bloch bands - the existence of period doubled Bloch bands [40]. These Bloch bands come into being if a periodic state - a Bloch state with $\kappa=0$ - becomes spatially unstable in a period doubling bifurcation, giving birth to a $4 \pi$-periodic state. This state is now again embedded into a (perioddoubled) Bloch band.

The period doubling bifurcation has already been introduced in Sec. 3.2 for the case of a variation of the potential strength $\lambda$. As shown on the left-hand side of Fig. 11, the antisymmetric $2 \pi$-periodic state bifurcates when $\lambda$ is increased above a critical value $\lambda_{\text {pd }}$. (We now use the index 'pd' for period doubling to distinguish it from the critical value for the emergence of looped Bloch bands).

The period-doubling bifurcation can also take place if the nonlinearity $g$ is increased while $\lambda$ and the normalization (4.3) remain fixed. This is illustrated on the left-hand side of Fig. 16, where we have plotted the chemical potential $\mu$ as a function of $g$ for the $2 \pi$-periodic state (green) and the $4 \pi$-periodic state (red). The wave functions of these two states are compared on the right-hand side of Fig. 16, for $g=1 / 8$.

The asymmetric $2 \pi$-periodic state is just the Bloch state with $\kappa=0$ in the first excited band which is shown in Fig. 17 (green line). Similarly, the $4 \pi$-periodic state is embedded in a 'period-doubled' Bloch band which is also shown in Fig. 17] as a red line.

The connection to the period-doubling bifurcation of an antisymmetric periodic state has several implications for the existence and stability of nonlinear Bloch states: Firstly, period-doubled Bloch bands obviously do not exist for $g=0$, they come into being when $g$ is increased over the critical value $g_{\text {pd }}$ for a period doubling bifurcation. Secondly, it was already argued in Sec. 3.2 that the corresponding $2 \pi$-periodic state becomes spatially unstable in this bifurcation. The lowest symmetric periodic state corresponding to the ground band is always unstable for $\lambda>0$, while the anti-symmetric state corresponding to the first excited band is is always unstable for $\lambda<0$ (cf. the stability map in Fig. 13).

This has several implications for the existence and stability of nonlinear Bloch states. 


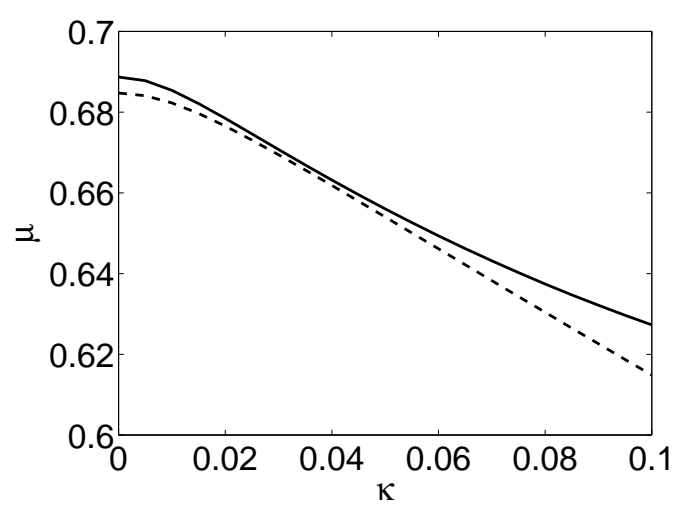

Figure 17. A $4 \pi$-periodic Bloch band (-) around $\kappa=0$ and the corresponding corresponding first excited $2 \pi$-periodic Bloch band $(---)$ for $\lambda=0.7$ and $g=1 / 8$.

The $4 \pi$-periodic states exist only after a bifurcation, i.e. for $g>g_{\mathrm{pd}}$. They are stable until the next bifurcation. As argued in Sec. 3.2, the corresponding $2 \pi$ periodic state becomes spatially unstable at this bifurcation.

\section{The Gaussian comb}

Finally we present some numerical results for a more realistic potential, consisting of a periodic structure of narrow Gaussian peaks,

$$
V(x)=\lambda \sum_{n} \frac{1}{\sqrt{2 \pi} \Delta x} \exp \left[-\frac{(x-2 \pi n)^{2}}{2 \Delta x^{2}}\right] .
$$

with $\Delta x=0.1$. The area of each Gaussian peak is normalized to unity. The paramters are such that $\Delta x \ll \xi \ll d$, where $\xi=1 / \sqrt{2 g \rho}$ is the healing length and $\rho$ is the averaged condensate density. This means that changes in the condensate density are 'healed' on a length scale which is much longer than the width of the Gaussian potential barriers, but smaller than the separation of the barriers. This gives a rough argument why the Gaussian comb is indeed well approximated by a delta-comb discussed above. Such smooth potentials can be implemented, at least in principle, in current atom-chip experiments (see [27] for a recent review, see also the discussion in [49]).

\subsection{Solutions of the time-independent NLSE}

We consider the solutions of the time-independent nonlinear Schrödinger equation in a spatial Poincaré section in phase space as in Sec. 2. The Figs. 18 and 19 show $\left(\psi_{n}, \psi_{n}^{\prime}\right)=$ $\left(\psi(2 \pi n), \psi^{\prime}(2 \pi n)\right)$ obtained by a numerical integration for different values of the potential strength $\lambda$. The dynamics in phase space strongly resembles the one for the delta-comb illustrated in the Figs. 4 and 5 .

Again we find trivial and symmetric periodic solutions, whose spatial stability properties are very similar to the case of a delta-comb. A pitchfork bifurcation of these periodic 

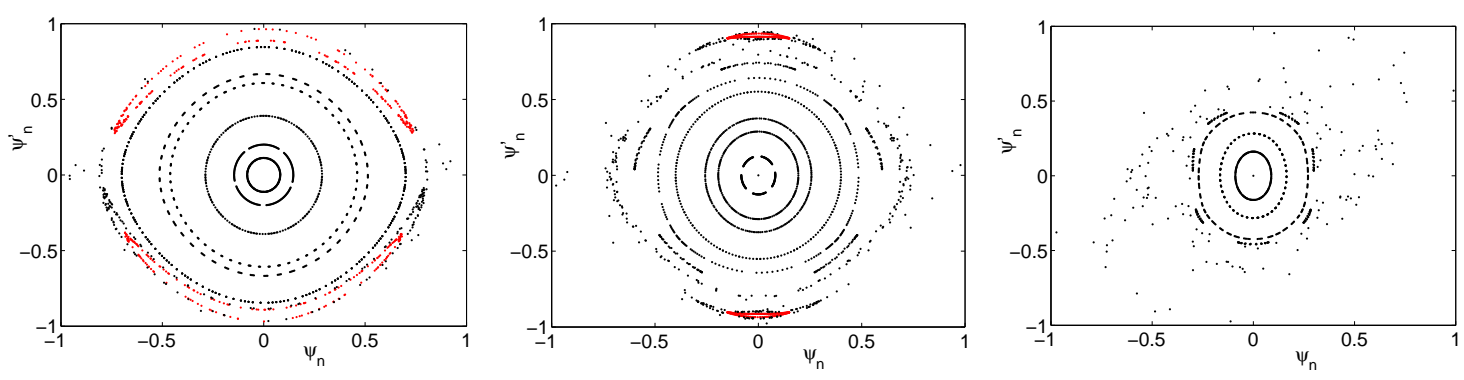

Figure 18. Poincaré section in phase space $\left(\psi_{n}, \psi_{n}^{\prime}\right)$ for the NLSE in a Gaussian comb with $g=1$, $\mu=1$ and $\lambda=0.02,0.1,0.5$ (from left to right).
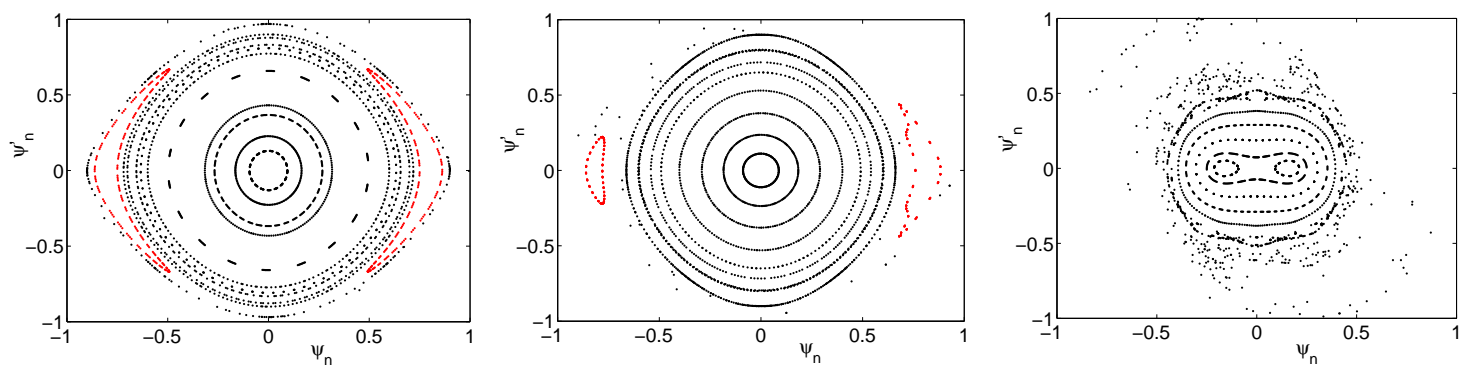

Figure 19. As figure 18, however for $\lambda=-0.02,-0.1,-0.5$ (from left to right).

solutions occurs when a control-parameter is varied. The bifurcation of the trivial periodic state for $\lambda>0$ is illustrated in the upper panel of Fig. 20. The lower panel shows a $4 \pi$-periodic wavefunction that emerges in the bifurcation.

\subsection{Dynamical (in)stability}

Up to now only the spatial stability of periodic solutions has been discussed, not considering the temporal behavior, induced by the time-dependent nonlinear Schrödinger equation

$$
\left(-\frac{1}{2} \frac{\partial^{2}}{\partial x^{2}}+V(x)+g|\psi(x, t)|^{2}\right) \psi(x, t)=\mathrm{i} \frac{\partial \psi}{\partial t} .
$$

In the following we analyze the temporal stability of the periodic solutions in the Gaussian comb discussed above. The time-dependent NLSE can lead to classical chaotic dynamics [61] and dynamical instability, which usually leads to a depletion of the Bose-condensed phase [15]. Related work on the dynamical stability of the NLSE in a periodic potentials can be found in $[8,9,12]$.

The energetical stability of a Bloch state $u_{\kappa}(x)$ can be calculated from perturbation theory $[70,71]$. We make the ansatz

$$
\psi(x)=\mathrm{e}^{\mathrm{i} \kappa x}\left[u_{\kappa}(x)+\delta u_{\kappa}(x)\right]
$$

with a small perturbation $\delta u_{\kappa}(x)$, that can be decomposed into different modes $\mathrm{e}^{ \pm \mathrm{i} q x}$. A Bloch state $u_{\kappa}(x)$ is energetically stable and hence represents a superflow, if it is a local 

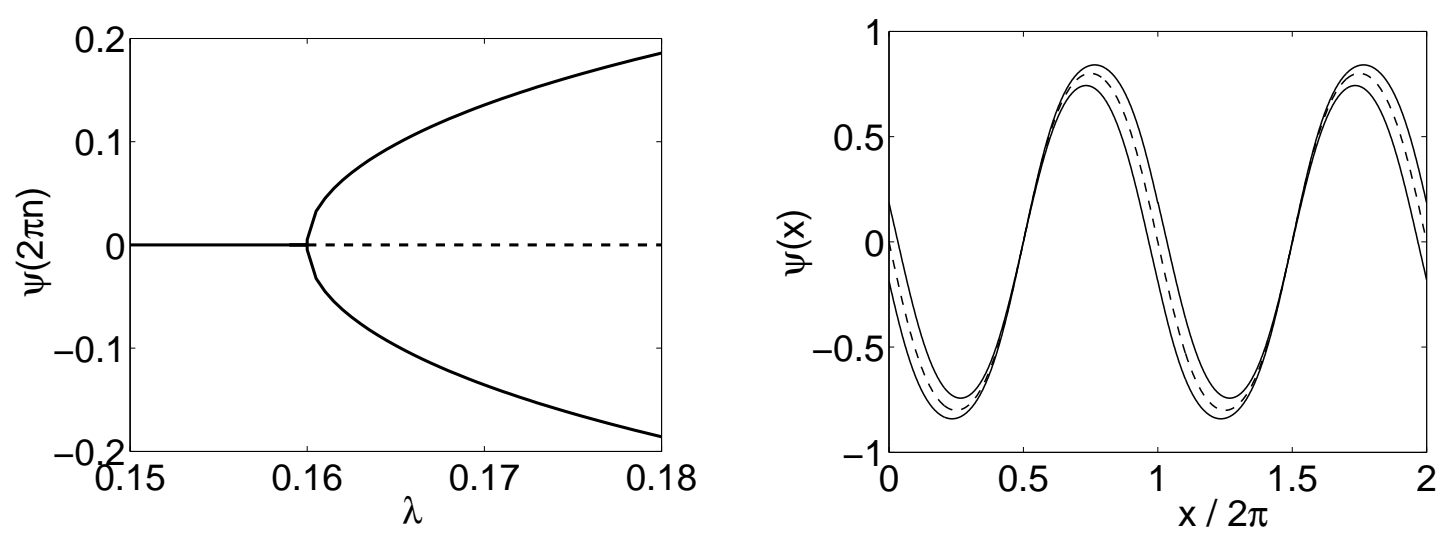

Figure 20. Upper panel: Bifurcation of the trivial periodic state in a repulsive Gaussian comb for $\mu=1$. Lower panel: The unstable $2 \pi$-periodic wave function (dashed line) in comparison with the stable $4 \pi$-periodic wave functions (solid lines) in a Gaussian comb with $\lambda=0.18$ and $\mu=1$.

minimum of the Hamiltonian

$$
H=\int \psi^{*}\left(-\frac{1}{2} \frac{\partial^{2}}{\partial x^{2}}+V(x)\right) \psi+\frac{g}{2}|\psi|^{4}-\mu|\psi|^{2} \mathrm{~d} x
$$

Otherwise a perturbation can lower the energy of the solution and the system becomes energetically unstable and superfluidity is lost, which is also called Landau-instability. Inserting the ansatz (5.3) into the Hamiltonian and neglecting higher order terms reveals that the Bloch state is energetically stable if the operator

$$
M(q)=\left(\begin{array}{cc}
L(\kappa+q) & g \psi^{2} \\
g \psi^{* 2} & L(-\kappa+q)
\end{array}\right)
$$

with

$$
L(q)=-\frac{1}{2}\left(\frac{\partial}{\partial x}+\mathrm{i} q\right)^{2}+V(x)+2 g|\psi|^{2}-\mu .
$$

is positive definite for all $-1 / 2 \leq q \leq 1 / 2$ [71].

Similarly one can deduce the dynamical stability by assuming a time-dependent perturbation

$$
\psi(x, t)=\mathrm{e}^{\mathrm{i} k x-\mathrm{i} \mu t}\left[u_{k}(x)+\delta u_{k}(x, t)\right] .
$$

The pertubation $\delta u_{\kappa}(x, t)$ grows exponentially in time if the operator $\sigma_{z} M(q)$ with $\sigma_{z}=$ $\operatorname{diag}(1,-1)$ has a complex eigenvalue for some $q$. Hence the Bloch state $u_{\kappa}(x)$ is dynamically stable only if the spectrum of $\sigma_{z} M(q)$ is purely real for all $-1 / 2 \leq q \leq 1 / 2$. Note that dynamical instability always implies energetical instability, as shown in [71].

Here we only consider strictly periodic states, which are Bloch states with $\kappa=0$. The eigenvalues $\zeta(q)$ of $\sigma_{z} M(q)$ are calculated for the periodic states discussed above in dependence of the potential strength $\lambda$. In Fig. 21 the maximum imaginary parts of the eigenvalues $\zeta(q)$ are plotted, where the maximum is taken over all $q \in[-1 / 2,1 / 2]$. One 

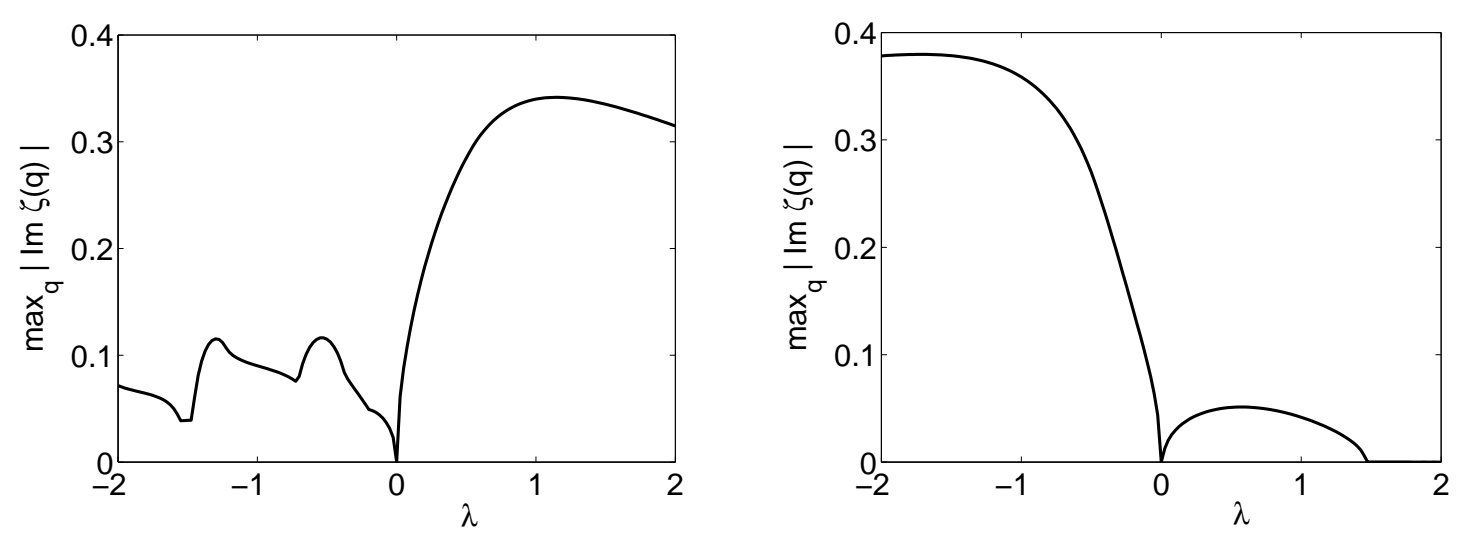

Figure 21. Eigenvalues of $\sigma_{z} M(q)$, indicating the dynamical (in)stability, for the anti-symmetric (left) and symmetric (right) periodic solutions in dependence of the potential strength $\lambda$. Shown is the maximum modulus of the imaginary parts of the eigenvalues of $\sigma_{z} M(q)$, where the maximum is taken over all eigenvalues and all $q$ in the first Brillouin zone $q \in(-0.5,0.5)$.

observes that the spectrum of $\sigma_{z} M(q)$ is never purely real for the antisymmetric periodic states (except for $\lambda=0$ ). Hence the these states are always dynamically and energetically unstable. The symmetric periodic state is dynamically unstable for attractive potentials and not too strong repulsive potentials. It is stabilized if the potential strength $\lambda$ exceeds a critical value $\lambda_{d}>0$. However it still is energetically unstable for all values of $\lambda$. Similar results are obtained for the delta-comb discussed in the previous sections.

The dynamical (in)stability is illustrated in Fig. 22, where the time evolution of the squared modulus $|\psi(x, t)|^{2}$ of a wavefunction is plotted. The initial state $\psi(x, t=0)$ is chosen as a symmetric periodic solution for $\lambda=1$ resp. $\lambda=2$ and $\mu=1$ plus a small random perturbation. According to the results from perturbation theory illustrated in Fig. 21, the system is dynamically unstable for $\lambda=1$ and dynamically stable for $\lambda=2$. This is well confirmed by the results of the wavepacket propagation. For $\lambda=1$ the onset of dynamical instability is clearly visible at $t \approx 150$, whereas no instability can be observed for $\lambda=2$.

Note that the temporal stability of the states is not affected at all by the spatial stability properties. The onset of dynamical instability is solely determined by the spectrum of $\sigma_{z} M(q)$ and the strength of the initial random perturbation. On the contrary one finds that the parameter values for which a periodic state is spatially or dynamically stable are quite different. The lowest symmetric state is spatially stable only for $\lambda_{c}<\lambda<0$ (cf. Fig. 12), whereas it is dynamically stable only for $\lambda>\lambda_{d}>0$. (cf. Fig. 21). One can even increase the potential strength $\lambda$ slowly through the critical value $\lambda_{c}$ for a bifurcation without affecting the onset of dynamical instability.

\section{Summary}

Summing up, the stationary solutions of the nonlinear Schrödinger equation for a deltacomb potential are analyzed. This somewhat artificial model system is of considerable interest since it allows analytical solutions in terms of Jacobi elliptic functions. 

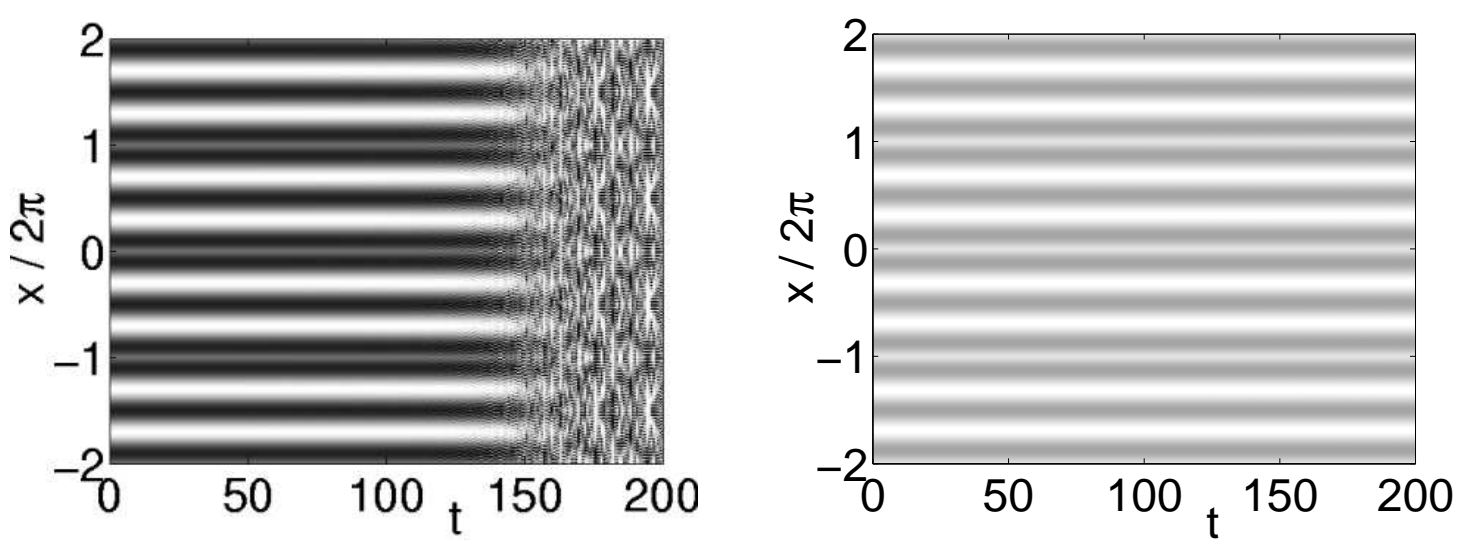

Figure 22. Time evolution of the squared modulus $|\psi(x, t)|^{2}$ of a wave function in a Gaussian comb of strength $\lambda=1$ (left) resp. $\lambda=2$ (right) The initial state $\psi(x, t=0)$ was chosen as the symmetric periodic solution for $\mu=1$ plus a small random perturbation.

The analogy to a classical nonlinear oscillator problem, the kicked nonlinear Hill equation is pointed out and the dynamics in phase space is discussed. It is shown that a repulsive nonlinearity typically leads to spatially chaotic dynamics and finally a divergence of the wave function, whereas the wave function always remains bounded for an attractive nonlinearity. Periodic solutions, which are fixed points of the phase space dynamics, are analyzed in detail. The fixed points are spatially stable only for certain parameter values, for which a stability map is calculated. The stability is lost in a Hamiltonian bifurcation scenario, giving rise to new period doubled fixed points.

Nonlinear Bloch bands are calculated for the delta-comb showing the celebrated loop structures. It is shown that the Bloch states with zero quasi momentum are just the periodic solutions mentioned above, which are given analytically in terms of Jacobi elliptic functions. The critical nonlinearity parameter for the emergence of loops is calculated analytically. Furthermore the emergence of $4 \pi$-periodic Bloch bands is discussed and linked to the period doubling bifurcation of periodic solutions mentioned above.

Finally an extension to a more realistic potential, a periodic arrangement of narrow Gaussian potential barriers, shows that the properties of the stationary solutions remain qualitatively the same as for the delta-comb. In addition the temporal stability of the periodic solutions is analyzed. Antisymmetric periodic solutions are always dynamically unstable, whereas the symmetric periodic solutions are stabilized if the potential strength exceeds a critical value $\lambda>\lambda_{d}>0$.

The properties of stationary states of the nonlinear Schrödinger equation are of fundamental interest for the study of BECs in optical lattices. For example the emergence of loops leads to dynamical instability of BECs in tilted optical lattices because an adiabatic evolution is no longer possible here $[25,39]$. But not all solutions in periodic potential must take the form of Bloch states, as it was shown in the present paper. In contrast, most solutions of the nonlinear Schrödinger equation with a repulsive potential will be spatially chaotic or even divergent. This deserves further studies. 


\section{Acknowledgments}

Support from the Deutsche Forschungsgemeinschaft via the Graduiertenkolleg "Nichtlineare Optik und Ultrakurzzeitphysik" and the research fellowship programme (grant number WI 3415/1) is gratefully acknowledged.

\section{References}

[1] Abramowitz, M., And Stegun, I. A. Handbook of Mathematical Functions. Dover Publications, Inc., New York, 1972.

[2] Adami, R., And Saccetti, A. The transition from diffusion to blow-up for a nonlinear Schrödinger equation in dimension 1. J. Phys. A 38 (2005), 8379.

[3] Aguiar, C. P. Malta, M. Baranger and K. T. R. Davis, M. Bifurcations of periodic trajectories in non-integrable hamiltonian systems with two degrees of freedom: Numerical and analytical results. Ann. Phys. (N.Y.) 180 (1987), 167.

[4] Albiez, M., Gati, R., Fölling, J., Hunsmann, S., Cristiani, M., and Oberthaler, M. K. Direct observation of tunneling and nonlinear self-trapping in a single bosonic Josephson junction. Phys. Rev. Lett. 95 (2005), 010402.

[5] Alfimov, G. L., And Zezyulin, D. A. Nonlinear modes for the Gross-Pitaevskii equation - a demonstrative computation approach. Nonlinearity 20 (2007), 2075.

[6] Bloch, I., Dalibard, J., And Zwerger, W. Many-body physics with ultracold gases. Rev. Mod. Phys. 80 (2008).

[7] Bowman, T. T. M. Bifurcation of solutions of nonlinear boundary value problem. Nonlin. Anal. Theory, Methods Appl. 5 (1981), 655.

[8] Bronski, J. C., Carr, L. D., Carretero-González, R., Deconinck, B., Kutz, J. N., And Promislow, K. Stability of attractive Bose-Einstein condensates in a periodic potential. Phys. Rev. E 64 (2001), 056615.

[9] Bronski, J. C., Carr, L. D., Deconinck, B., and Kutz, J. N. Bose-Einstein condensates in standing waves: The cubic nonlinear Schrödinger equation with a periodic potential. Phys. Rev. Lett. 86 (2001), 1402.

[10] Burger, S., Bongs, K., Dettmer, S., Ertmer, W., Sengstock, K., SanPera, A., Shlyapnikov, G., And Lewenstein, M. Dark solitons in Bose-Einstein condensates. Phys. Rev. Lett. 83 (1999), 5198.

[11] Carr, L. D., Clark, C. W., and Reinhardt, W. P. Stationary solutions of the one-dimensional nonlinear Schrödinger equation. Phys. Rev. A 62 (2000), 063610, 063611 .

[12] Carr, L. D., Kutz, J. N., and Reinhardt, W. P. Stability of stationary states in the cubic nonlinear Schrödinger equation: Applications to Bose-Einstein condensates. Phys. Rev. E 63 (2001), 066604. 
[13] Carretero-González, R., Frantzeskakis, D. J., and Kevrekidis, P. G. Nonlinear waves in Bose-Einstein condensates: physical relevance and mathematical techniques. Nonlinearity 21 (2008), R139.

[14] Cartarius, H., Main, J., And Wunner, G. Discovery of exceptional points in a Bose-Einstein condensation of gases with attractive 1/r-interaction. Phys. Rev. A 7 r (2008), 013618.

[15] Castin, Y., And Dum, R. Instability and depletion of an excited Bose-Einstein condensate in a trap. Phys. Rev. Lett. 79 (1997), 3553.

[16] Castin, Y., And Dum, R. Low-temperature Bose-Einstein condensates in timedependent traps: Beyond the $u(1)$ symmetry-breaking approach. Phys. Rev. A 57 (1998), 3008.

[17] Cervero, J. M. Pt-symmetry in one-dimensional quantum periodic potentials. Phys. Lett. A 317 (2003), 26.

[18] Cervero, J. M., And Rodriguez, A. The band spectrum of periodic potentials with pt-symmetry. J. Phys. A 37 (2004), 10167.

[19] Chirikov, B. V. Universal instability of many-dimensional oscillator systems. Phys. Rep. 52 (1979), 263.

[20] Coz, S. L., Fukuizumi, R., G, F., Ksherim, B., and Y.Sivan. Instability of bound states of a nonlinear Schrödinger equation with a Dirac potential. Physica $D$ 38 (2008), 8379.

[21] D'Agosta, R., Malomed, B. A., and Presilla, C. Stationary solutions of the Gross-Pitaevskii equation with a linear counterpart. Phys. Lett. A 275 (2000), 424.

[22] Danshita, I., And Tsuchiya, S. Stability of Bose-Einstein condensates in a KronigPenney potential. Physical Review A 75 (2007), 033612.

[23] Dodd, R. K., Eilbeck, J. C., Gibbon, J. D., And Morris, H. C. Solitons and nonlinear wave equations. Academic Press, London, 1982.

[24] Eiermann, B., Anker, T., Albie, M., Taglieber, M., Treutlein, P., MarZlin, K.-P., And Oberthaler, M. K. Bright Bose-Einstein gap solitons of atoms with repulsive interaction. Phys. Rev. Lett. 92 (2004).

[25] Fallani, L., Sarlo, L. D., Lye, J. E., Modugno, M., Sears, R., Fort, C., And InGUSCIO, M. Observation of dynamical instability for a Bose-Einstein condensate in a moving 1d optical lattice. Phys. Rev. Lett. 93 (2004), 140406.

[26] Fibich, G., Sivan, Y., And Weinstein, M. I. Bound states of nonlinear Schrödinger equations with a periodic nonlinear microstructure. Physica $D 217$ (2006), 31.

[27] Fortagh, J., And Zimmermann, C. Magnetic microtraps for ultracold atoms. Rev. Mod. Phys. 79 (2007), 235. 
[28] Graefe, E. M., And Korsch, H. J. Crossing scenario for a nonlinear non-hermitian two-level system. Czech. J. Phys. 56 (2006), 1007.

[29] Greiner, M., Bloch, I., Mandel, O., Hänsch, T. W., and Esslinger, T. Bose-Einstein condensates in 1d- and 2d- optical lattices. Appl. Phys. B 73 (2001), 769 .

[30] Griffiths, D. J., And Taussig, N. F. Scattering from a locally periodic potential. Am. J. Phys. 60 (1992), 883.

[31] Hsu, C. S. Some simple exact periodic responses for a nonlinear system under parametric excitation. J. Appl. Mech. 42 (1974), 176.

[32] Jackson, R. K., And Weinstein, M. I. Geometric analysis of bifurcation and symmetry breaking in a Gross-Pitaevskii equation. J. Stat. Phys. 116 (2004), 881.

[33] Jona-lasinio, M., Morsch, O., Cristiani, M., Malossi, N., Müller, J. H., Courtade, E., Anderlini, M., And Arimondo, E. Asymmetric Landau-Zener tunneling in a periodic potential. Phys. Rev. Lett. 91 (2003), 230406.

[34] Khaykovich, L., Schreck, F., Ferrari, G., Bourdel, T., Cubizolles, J., Carr, L. D., Castin, Y., And SAlomon, C. Formation of a matter-wave bright soliton. Science 296 (2002).

[35] Kominis, Y. Analytical solitary wave solutions of the nonlinear Kronig-Penney model in photonic structures. Phys. Rev. E 73 (2006), 066619.

[36] Korsch, H. J., Jodl, H.-J., And Hartmann, T. Chaos - A Program Collection for the PC, 3nd Ed. Springer-Verlag, Heidelberg, New-York, 2008.

[37] Lawden, D. F. Elliptic Functions and Applications. Springer, New York, 1989.

[38] Li, W. D., And Smerzi, A. Nonlinear Kronig-Penney model. Phys. Rev. E 70 (2004), 016605.

[39] Liu, J., Wu, B., And Niu, Q. Nonlinear evolution of quantum states in the adiabatic regime. Phys. Rev. Lett. 90 (2003), 170404.

[40] Machholm, M., Nicolin, A., Pethick, C. J., And Smith, H. Spatial period doubling in Bose-Einstein condensates in an optical lattice. Phys. Rev. A 69 (2004), 043604 .

[41] Makris, K. G., El-Ganainy, R., Christodoulides, D. N., and Musslimani, Z. H. Beam dynamics in pt symmetric optical lattices. Phys. Rev. Lett. 100 (2008), 103904 .

[42] Meyer, K. R. Generic bifurcations of periodic points. Trans. AMS 149 (1970), 95.

[43] Month, L. A., And Rand, R. H. Bifurcation of 4:1 subharmonics in the nonlinear Mathieu equation. Mechanics Research Comm. 9 (1982), 233.

[44] Moon, F. C. Chaotic Vibrations. John Wiley, New York, 1987. 
[45] Morsch, O., and Oberthaler, M. Dynamics of Bose-Einstein condensates in optical lattices. Rev. Mod. Phys. 78 (2006), 179.

[46] Musslimani, Z. H., Makris, K. G., El-Ganainy, R., and Christodoulides, D. N. Optical solitons in pt periodic potentials. Phys. Rev. Lett. 100 (2008), 030402.

[47] N̈̈GERL, H.-C. private communication.

[48] Nayfeh, A. H., And Mook, D. T. Nonlinear Oscillations. John Wiley, New York, 1979.

[49] Paul, T., Richter, K., And Schlagheck, P. Nonlinear resonant transport of Bose-Einstein condensates. Phys. Rev. Lett. 94 (2005), 020404.

[50] Paul, T., Schlagheck, P., Leboeuf, P., and Pavloff, N. Superfluidity versus Anderson localization in a dilute Bose gas. Phys. Rev. Lett. 98 (2007), 210602.

[51] Pethick, C. J., And Smith, H. Bose-Einstein Condensation in Dilute Gases. Cambridge University Press, Cambridge, 2002.

[52] Pitaevskit, L., And Stringari, S. Bose-Einstein Condensation. Oxford University Press, Oxford, 2003.

[53] Porter, M. A., And Cvitanovic, P. A perturbative analysis of modulated amplitude waves in Bose-Einstein condensates. Chaos 14 (2004), 739.

[54] Rapedius, K., And Korsch, H. J. Resonance solutions of the nonlinear schrödinger equation in an open double-well potential. J. Phys. B (2009), in press, e-print arxiv: 0809.2748.

[55] Rapedius, K., Witthaut, D., And Korsch, H. J. Analytical study of resonant transport of Bose-Einstein condensates. Phys. Rev. A 73 (2006), 033608.

[56] Saccetti, A. Spectral splitting method for nonlinear Schrödinger equations with singular potential. J. Chem. Phys. 227 (2007), 1483.

[57] Seaman, B. T., Carr, L. D., and Holland, M. J. Nonlinear band structure in Bose-Einstein condensates: Nonlinear Schrödinger equation with a Kronig-Penney potential. Phys. Rev. A 71 (2005), 033622.

[58] Seaman, B. T., Carr, L. D., and Holland, M. J. Period doubling, two-color lattices, and the growth of swallowtails in Bose-Einstein condensates. Phys. Rev. A 72 (2005), 033602.

[59] Taras-Semchuk, D., And Gunn, J. M. F. Superfluid flow past an array of scatterers. Phys. Rev. B 60 (1999), 13139.

[60] Theodorakis, S., And Leontidis, E. Bound states in a nonlinear Kronig-Penney model. J. Phys. A 30 (1997), 4835.

[61] Thommen, Q., Garreau, J. C., and Zehnlé, V. Classical chaos with BoseEinstein condensates in tilted optical lattices. Phys. Rev. Lett. 91 (2003), 210405. 
[62] Trimborn, F., Witthaut, D., And Korsch, H. J. Beyond mean-field dynamics of small Bose-Hubbard systems based on the number-conserving phase space approach. Phys. Rev. A 79 (2009), 013608.

[63] Trimborn, F., Witthaut, D., and Wimberger, S. Mean-field dynamics of a two-mode Bose-Einstein condensate subject to noise and dissipation. J. Phys. B 41 (2008), 171001.

[64] Wan, Y., And Soukoulis, C. M. One-dimensional nonlinear Schrödinger equation: A nonlinear dynamical approach. Phys. Rev. A 41 (1990), 800.

[65] Witthaut, D., Graefe, E. M., and Korsch, H. J. Towards a Landau-Zener formula for an interacting Bose-Einstein condensate in a two-level system. Phys. Rev. A 73 (2006), 063609.

[66] Witthaut, D., Graefe, E. M., Wimberger, S., and Korsch, H. J. BoseEinstein condensates in accelerated double-periodic optical lattices: coupling and crossing of resonances. Phys. Rev. A 75 (2007), 013617.

[67] Witthaut, D., Mossmann, S., And Korsch, H. J. Bound and resonance states of the nonlinear Schrödinger equation in simple model systems. J. Phys. A 38 (2005), 1777 .

[68] Witthaut, D., Werder, M., Mossmann, S., And Korsch, H. J. Blochoscillations of Bose-Einstein condensates: Breakdown and revival. Phys. Rev. E 71 (2005), 036625.

[69] Wu, B., And Niu, Q. Nonlinear Landau-Zener tunneling. Phys. Rev. A 61 (2000), 023402 .

[70] Wu, B., AND Niu, Q. Landau and dynamical instabilities of the superflow of BoseEinstein condensates in optical lattices. Phys. Rev. A 64 (2001), 061603.

[71] Wu, B., And Niu, Q. Superfluidity of Bose-Einstein condensates in an optical lattice: Landau-Zener tunneling and dynamical instability. New J. Phys. 5 (2003), 104. 